\title{
Extracellular matrix protein laminin $\beta 1$ regulates pain sensitivity and anxiodepression-like behaviors in mice
}

\author{
Zhen-Zhen Li, ${ }^{1,2}$ Wen-Juan Han, ${ }^{1}$ Zhi-Chuan Sun, ${ }^{1}$ Yun Chen, ${ }^{3}$ Jun-Yi Sun, ${ }^{3}$ Guo-Hong Cai, ${ }^{1}$ Wan-Neng Liu, ${ }^{1,4}$ Tao-Zhi Wang, ${ }^{1,5}$ \\ Yang-Dan Xie, ${ }^{3}$ Hong-Hui Mao, ${ }^{1}$ Fei Wang, ${ }^{1,6}$ Sui-Bin Ma, ${ }^{1}$ Fu-Dong Wang, ${ }^{1}$ Rou-Gang Xie, ${ }^{1}$ Sheng-Xi Wu, ${ }^{1}$ and Ceng Luo \\ 'Department of Neurobiology, School of Basic Medicine, '2Department of Neurosurgery, Xijing Hospital, and ${ }^{3}$ The Second Regiment, School of Basic Medicine, Fourth Military Medical University, Xi'an, China.

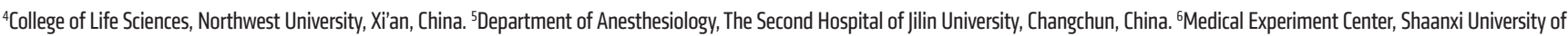 \\ Chinese Medicine, China.
}

\begin{abstract}
Patients with neuropathic pain often experience comorbid psychiatric disorders. Cellular plasticity in the anterior cingulate cortex (ACC) is assumed to be a critical interface for pain perception and emotion. However, substantial efforts have thus far been focused on the intracellular mechanisms of plasticity rather than the extracellular alterations that might trigger and facilitate intracellular changes. Laminin, a key element of the extracellular matrix (ECM), consists of one $\alpha-$, one $\beta-$, and one $\gamma$-chain and is implicated in several pathophysiological processes. Here, we showed in mice that laminin $\beta 1$ (LAMB1) in the ACC was significantly downregulated upon peripheral neuropathy. Knockdown of LAMB1 in the ACC exacerbated pain sensitivity and induced anxiety and depression. Mechanistic analysis revealed that loss of LAMB1 caused actin dysregulation via interaction with integrin $\beta 1$ and the subsequent Src-dependent RhoA/LIMK/cofilin pathway, leading to increased presynaptic transmitter release probability and abnormal postsynaptic spine remodeling, which in turn orchestrated the structural and functional plasticity of pyramidal neurons and eventually resulted in pain hypersensitivity and anxiodepression. This study sheds new light on the functional capability of ECM LAMB1 in modulating pain plasticity and identifies a mechanism that conveys extracellular alterations to intracellular plasticity. Moreover, we identified cingulate LAMB1/integrin $\beta 1$ signaling as a promising therapeutic target for the treatment of neuropathic pain and associated anxiodepression.
\end{abstract}

\section{Introduction}

Neuropathic pain is frequently comorbid with psychiatric disorders, such as anxiety and depression, rendering it more resistant to classical treatment (1-4). In particular, aberrant psychiatric conditions may lead to an exaggerated duration and intensity of pain and drive a vicious cycle of pain and emotional aversion $(2,5,6)$. Thus, development of a new and effective treatment for comorbid psychiatric disorders in neuropathic pain remains a major challenge (7).

Functional imaging and experimental evidence have implicated the anterior cingulate cortex (ACC) in sensory perception and emotional responses (8-11). Neurons in the ACC are activated by nerve injury, and inhibition of central plasticity in the ACC produces analgesic as well as anxiolytic and antidepressive effects (12-17). Despite these advances, mounting efforts have thus far been focused on intracellular mechanisms of plasticity rather than the extracellular alterations that might trigger and facilitate intracellular changes in these cellular functions. The extracellular matrix (ECM) is known to provide not only a supporting scaffold for cells but also a unique class of first messengers acting on the adhesion receptors, which in turn can modulate cell functions in response to environmental changes, i.e., nox-

Authorship note: ZZL, WJH, and ZCS contributed equally to this work. Conflict of interest: The authors have declared that no conflict of interest exists. Copyright: () 2021, American Society for Clinical Investigation.

Submitted: November 24, 2020; Accepted: June 16, 2021; Published: June 22, 2021 Reference information: J Clin Invest. 2021;131(15):e146323.

https://doi.org/10.1172/JCl146323. ious challenges (18). The ECM in the CNS is associated with the regulation of synaptic plasticity and various pathophysiological processes (18). For example, in a mouse model of chronic pain, nerve injury alters the ECM microarchitecture and decreases ECM rigidity in the hippocampus, which is linked to cognitive defects in neuropathic pain (19). Given the role of the ACC as a critical interface for pain perception and emotion, we were therefore interested in knowing whether this structural plasticity of the ECM occurs in the ACC upon nerve injury. If so, what is the biochemical basis for this cingulate ECM plasticity? And how would this extracellular ECM abnormality facilitate the intracellular signal transduction pathway in the ACC and further lead to neuropathic pain and associated anxiodepression?

Laminins, which are key ECM elements, are a family of heterotrimeric glycoproteins consisting of one $\alpha$-, one $\beta$-, and one $\gamma$ chain. In mammals, 11 laminin chains ( $\alpha 1-5, \beta 1-3$, and $\gamma 1-3)$ and 16 combinations of these have been identified, with $\beta 1$ incorporated in 6 combinations $(20,21)$. The dual roles of laminins in tissue architecture and cell signaling make laminins well suited to convey information from extracellular to intracellular in threedimensional complexity. Laminins are widely distributed in the CNS and basement membrane of blood vessels. Further studies with endogenous expression profile and transgenic mice overexpressing LAMB1 show that LAMB1 is highly expressed in the cortex and associated with several psychiatric disorders $(22,23)$. For instance, prolonged stress decreases laminins expression in the frontal cortex and hippocampus (24). In patients with depression, laminin expression is decreased in the parieto-occipital cor- 
tex, and antidepressant treatment reverses this effect (25). On the other hand, higher laminin levels are found in the frontal cortex and hippocampus in patients with Alzheimer's disease, whereas spatial training in rats decreases LAMB1 expression in the hippocampus $(26,27)$. In contrast, little is known about whether and how laminins participate in the neuropathic pain and associated anxiodepression, and if so, which extracellular-intracellular interacting processes underlie these actions.

By using a combination of RNA-Seq, behavioral, biochemical, electrophysiological as well as fiber photometry methods, we uncovered a crucial role of LAMB1 in the ACC in the neuropathic pain and associated anxiodepression. Peripheral neuropathy downregulates LAMB1 expression in the ACC. Knockdown of ACC LAMB1 exacerbates pain sensitivity and induces anxiety and depression-like behaviors. Further mechanistic analysis revealed that loss of LAMB1 in the ACC after nerve injury causes actin dysregulation via interaction with integrin $\beta 1$ and the subsequent Src-dependent RhoA/LIMK/cofilin pathway, leading to increased presynaptic transmitter release probability and abnormal postsynaptic spine remodeling, which in turn orchestrates the structural and functional plasticity of pyramidal neurons and eventually results in pain hypersensitivity and anxiodepression. We believe this study sheds new light on the functional capability of ECM LAMB1 in modulating neuropathic pain and related anxiodepression and reveals a mechanism that conveys extracellular alterations to intracellular plasticity. Moreover, we have identified LAMB1/integrin $\beta 1$ signaling in the ACC as a potential therapeutic target for the treatment of neuropathic pain and associated anxiodepression.

\section{Results}

Peripheral neuropathy decreases LAMB1 expression in the ACC. Following spared nerve injury (SNI), mice developed long-lasting exaggerated pain responses, i.e., mechanical allodynia and thermal hyperalgesia (Supplemental Figure 1, A-J; supplemental material available online with this article; https://doi.org/10.1172/ JCI146323DS1), as well as anxiety and depression (Supplemental Figure 2, A-Q). To identify the genes that are involved in the pathological process of neuropathic pain and associated anxiodepression, we analyzed the contralateral ACC by RNA-Seq on day 56 after SNI, when stable pain hypersensitivity and aversive emotion were fully established (Supplemental Figure 3, A-G). We detected a total of 565 differentially expressed genes (DEGs), with 418 (74\%) genes upregulated and 147 (26\%) genes downregulated (Figure 1A). Kyoto Encyclopedia of Genes and Genomes (KEGG) analysis revealed significant enrichment of genes in various pathways, especially those for ECM-receptor interaction and focal adhesion (Figure 1B). Among ECM-related pathway genes, we identified 5 downregulated and 12 upregulated genes (Figure 1C). In particular, LAMB1, an ECM component and previously known to be involved in several physiopathological processes, showed significant transcriptional downregulation following SNI (Figure 1C). We further verified this at the mRNA and protein levels by quantitative real-time PCR (qRT-PCR) and Western blotting in the contralateral ACC at different time points after SNI, with significant downregulation of LAMB1 observed on days 28-56 after SNI (Figure 1, D and E).
We then characterized the expression profile of LAMB1 in the ACC. Using immunofluorescence staining, we detected broad expression of LAMB1 in the ACC. Double-immunofluorescence staining revealed that LAMB1 immunoreactivity was highly coexpressed with neuronal nuclear antigen (NeuN) and sparsely coexpressed with either glial fibrillary acidic protein (GFAP) or ionized calcium-binding adapter molecule 1 (Iba1) (Figure 2, A and B). Notably, we detected LAMB1 expression in both calcium/calmodulin-dependent protein kinase II-expressing (CaMKII-expressing) excitatory and glutamate decarboxylase 1-expressing (GAD67-expressing) inhibitory neurons (Figure 2, C and D). Together, these results suggest a potential link between ACC LAMB1 and neuropathic pain as well as pain-related anxiodepression.

Knockdown of LAMB1 in the ACC induces pain hypersensitivity and anxiodepression. To address the question of whether there is a causal relationship between activity-dependent changes in LAMB1 and neuropathic pain and anxiodepressive consequences, we generated recombinant adeno-associated virus 2/9 (AAV2/9) expressing a shRNA targeted against both LAMB1 and EGFP (AAV-shLamb1; Supplemental Figure 4A). Among the 3 strands of Lamb1 shRNA constructed, Lamb1 shRNA-1 showed the most prominent knockdown efficiency in the cultured cortical neurons (Supplemental Figure 4, B and C) and was hence chosen for intraACC injection in the subsequent experiments. We first assessed how loss of unilateral cingulate LAMB1 affects behavioral correlates of sensitization in pain pathways (the experimental schematic paradigm is shown in Supplemental Figure 4D). The efficiency of LAMB1 knockdown confined to the ipsilateral ACC was confirmed accordingly (Figure 3, A-C, and Supplemental Figure 4, E-H). Compared with mice expressing scrambled shRNA, mice expressing shLamb1 in the right ACC had a significantly greater response to von Frey hairs in the left, but not the right, hindpaw under the sham condition (Figure 3D and Supplemental Figure $5 \mathrm{~A})$. Twelve weeks after SNI surgery in the left hind limb, expression of AAV-shLamb1 in the right ACC led to exaggerated mechanical allodynia in the bilateral hind paws compared with scrambled shRNA (Figure 3E and Supplemental Figure 5B). In contrast, basal nociception of thermal stimuli and thermal hyperalgesia following SNI in bilateral hind paws were unaltered by knockdown of LAMB1 in the ACC (Figure 3F and Supplemental Figure 5C).

Neuropathic pain is frequently associated with psychiatric disorders, such as anxiety and depression (1-4). We next examined whether LAMB1 in the ACC influences neuropathic pain-related anxiety and depression according to several behavioral paradigms. In the elevated plus maze (EPM) test, sham-treated mice expressing shLamb1 traveled a shorter distance in the open arm compared with mice expressing scrambled shRNA (Figure 3G and Supplemental Figure 5, D and E). We observed no significant differences in distances traveled in the open arm 12 weeks after SNI between mice in 2 virion-injected groups, which might have been due to a ceiling effect after SNI (Figure 3G and Supplemental Figure 5, D and E). In the tail suspension test (TST), expression of AAV-shLamb1 resulted in longer immobility in sham-treated mice compared with AAV-scrambled shRNA-treated mice (Figure $3 \mathrm{H}$ ). The sucrose preference test (SPT) also revealed a significant reduction in the preference for sucrose after knockdown of LAMB1 in the ACC (Figure 3I). Thus, it can be inferred from the 
A

Sham vs. SNI volcano plot

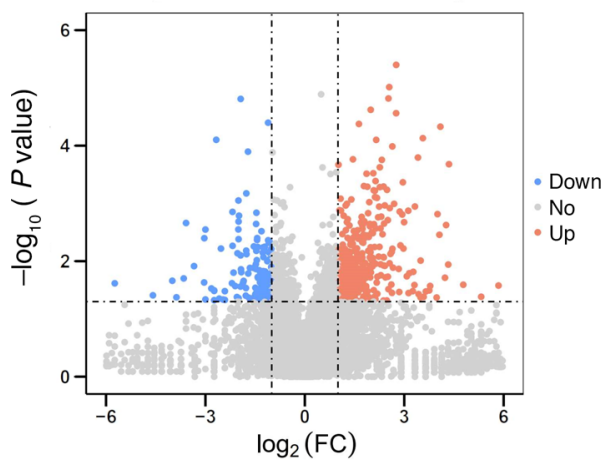

B

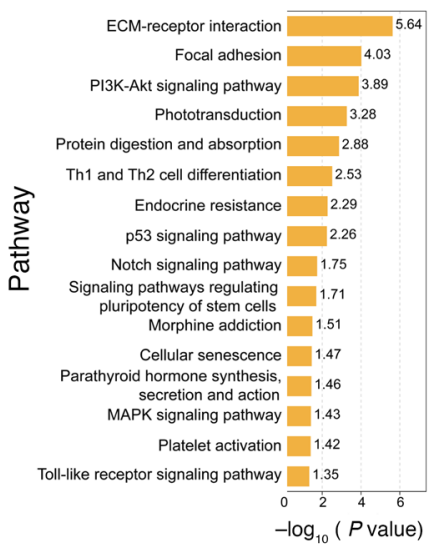

C

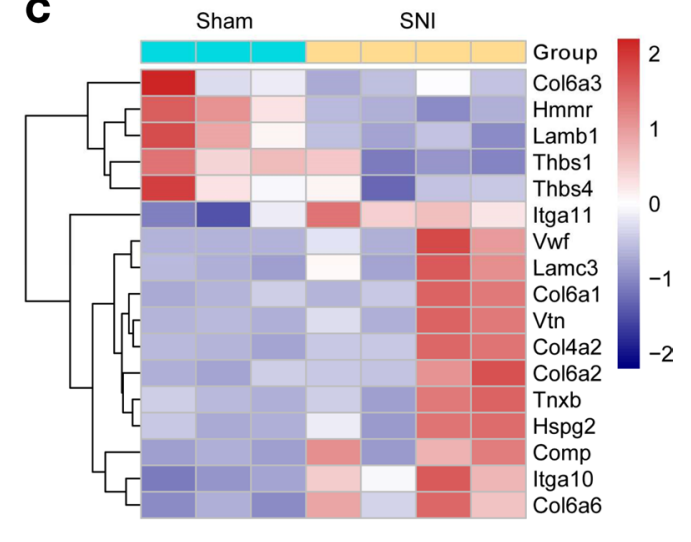

D

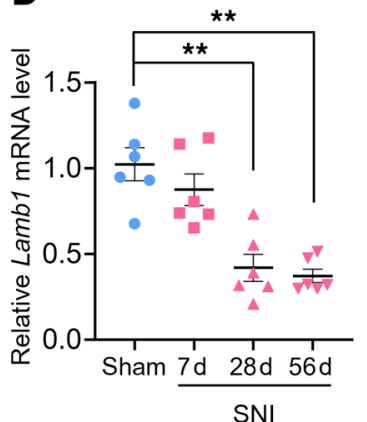

E

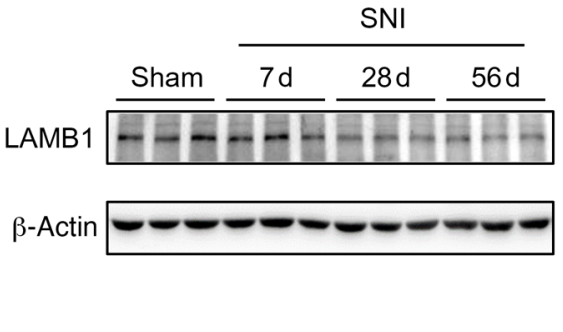

Figure 1. Peripheral neuropathy decreases LAMB1 expression in the ACC. (A) Volcano plot showing RNA-Seq data for contralateral ACC from SNI- versus sham-treated mice. DEGs are designated in orange (upregulation [up]) and blue (downregulation [down]) and defined as having an FDR of less than 0.05. FC, fold change. (B) Bar plot showing significant enrichment of DEGs in various pathways. (C) Relative expression levels are shown for genes in the ECMreceptor interaction pathway upon SNI as compared with sham treatment ( $n=3-4$ mice per group). ( $D$ and E) LAMB1 was downregulated in the ACC at both the mRNA (D) $(n=6)$ and protein $(\mathbf{E})(n=5)$ levels 7 days $(7 \mathrm{~d}), 28$ days $(28 \mathrm{~d})$, and 56 days $(56 \mathrm{~d})$ after SNI surgery. ${ }^{* *} P<0.01$, by Kruskal-Wallis $H$ test with Nemenyi's multiple-comparison test. Data are presented as the mean \pm SEM. See Supplemental Table 2 for detailed statistical information.

above observations that cingulate LAMB1 exerts a negative regulation of neuropathic pain and pain-related anxiodepression.

LAMB1 deficiency in the ACC orchestrates structural and functional plasticity of pyramidal neurons. How does ACC LAMB1 modulate neuropathic pain and the related anxiodepressive consequences? Structural and functional synaptic plasticity in the ACC is assumed to be a cellular basis for neuropathic pain and associated anxiodepression $(13,14,16,17)$. ECM molecules, including LAMB1, have been linked to the synapse stabilization, plasticity, and metaplasticity involved in psychiatric disorders (18, 27-29). Thus, we sought to determine whether silencing LAMB1 would induce structural and functional changes in ACC pyramidal neurons (experimental schematic paradigm is shown in Supplemental Figure 6A). First, we examined dendritic processes and spines of ACC pyramidal neurons using Golgi staining methods in mice expressing shLamb1 and scrambled shRNA. Sholl analysis revealed that the dendritic complexity of apical and basal dendrites of pyramidal neurons was increased in sham condition after LAMB1 knockdown, with no significant changes observed in the SNI condition (Figure 4, A-C). Furthermore, the densities of total apical spines were significantly higher in the pyramidal neurons of mice expressing shLamb1 than of sham-treated mice expressing scrambled shRNA (Figure 4, D and E). In parallel, the length of total apical spines of pyramidal neurons became much shorter after LAMB1 knockdown (Figure 4, D and F). High-resolution analysis enabled the classification of synaptic spines into stubby, mushroom, long/thin, and filopodia subtypes. Selective knockdown of LAMB1 in the ACC increased the density of stubby- and mushroom-shaped apical spines, with no obvious effect on long, thin and filopodia-like apical spines in sham-treated mice (Figure 4G). LAMB1 knockdown did not further increase the densities of stubby- or mushroom-shaped apical spines in SNI-treated mice, which might have been due to the post-SNI ceiling effect (Figure 4G). Unlike apical dendrites, the total density and length of basal spines were not influenced by ACC LAMB1 knockdown (Supplemental Figure 6, B-D). However, LAMB1 knockdown caused significant decreases in the density of stubby spines and increases in the densities of mushroom and filopodia spines of basal dendrites after SNI (Supplemental Figure 6E). The density of filopodialike basal spines was reduced by LAMB1 knockdown under the sham conditions (Supplemental Figure 6E). These results confirm that LAMB1 contributes to maintenance of the stabilization of synaptic spines in pyramidal neurons of the ACC and that disruption of LAMB1 induces abnormal spine remodeling.

Second, we assessed the functional consequences of LAMB1 deficiency on synaptic transmission in the ACC by using wholecell patch-clamp recording of pyramidal neurons (Figure $5 \mathrm{~A}$ ), 

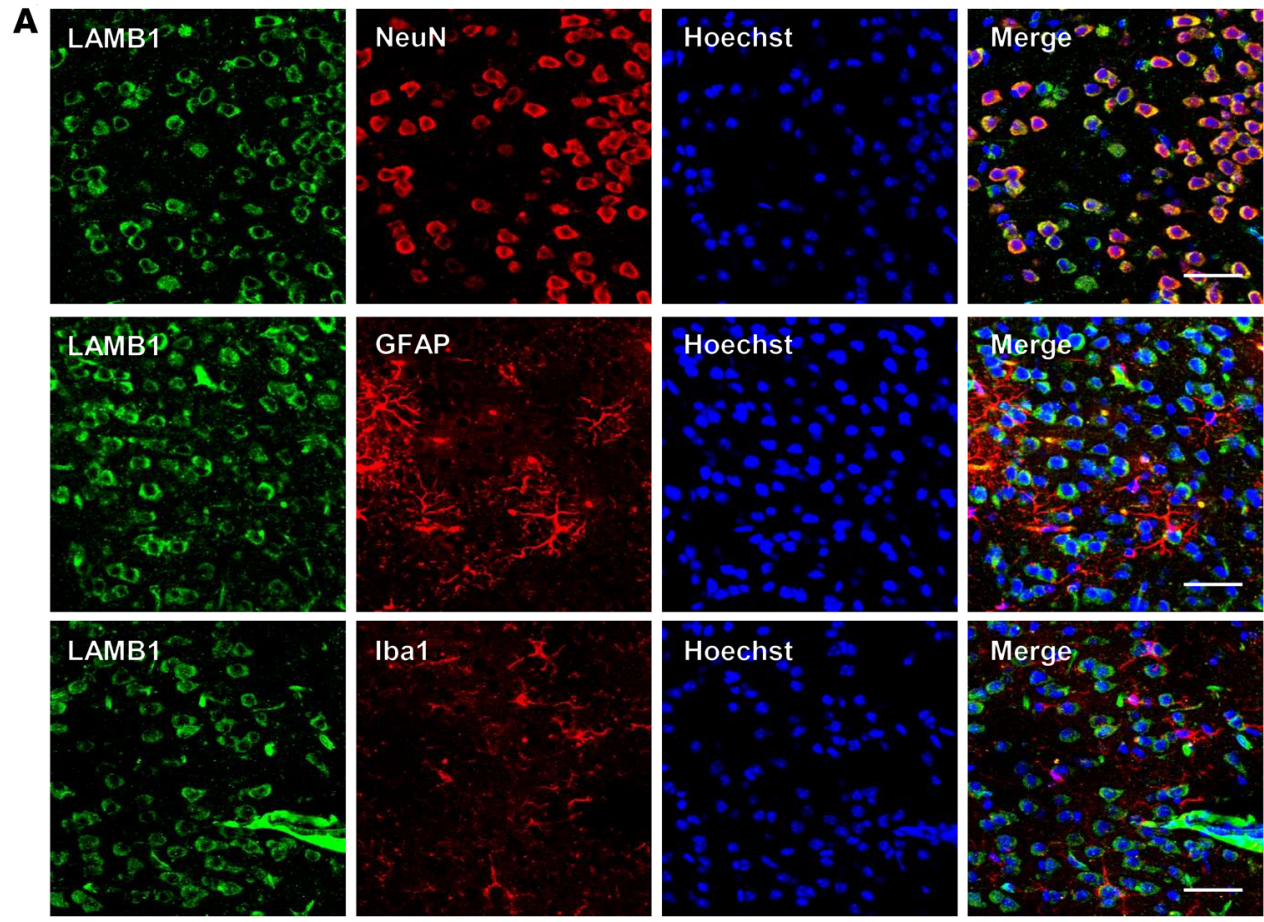

B

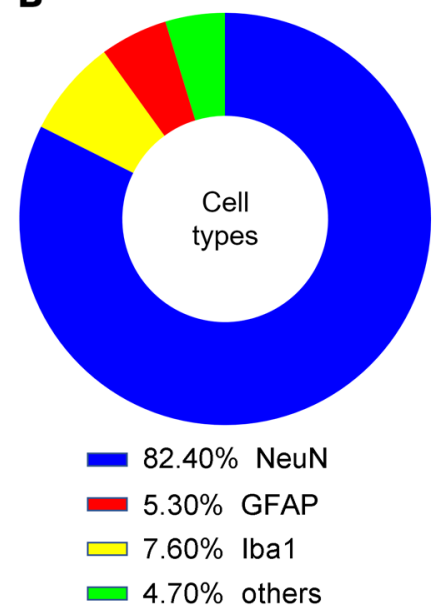

D

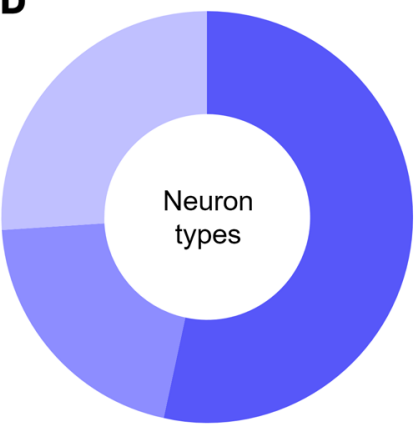

- $53.40 \%$ CaMK II

$\square 20.60 \%$ GAD67

$\square 26.00 \%$ Other types

Figure 2. Characterization of expression profile of LAMB1 in the ACC. (A and B) Representative immunofluorescence images (A) and quantitative summary (B) showing that LAMB1 was highly coexpressed with NeuN and sparsely coexpressed with GFAP or Iba1 $(n=3)$. (C and D) Representative immunofluorescence images $(\mathbf{C})$ and quantitative summary $(\mathbf{D})$ showing that LAMB1 expression was found in CaMKII+ neurons and GAD67 ${ }^{+}$neurons $(n=3)$. Scale bars: $30 \mu \mathrm{m}$ (A and $\mathbf{C}$ ).

which were identified on the basis of their morphological properties and ability to show spike frequency adaptation in response to prolonged depolarizing current injections (30). Three weeks after infection with AAV-shLamb1, we found that the excitability of ACC pyramidal neurons was significantly enhanced compared with scrambled shRNA-expressing neurons (Figure 5B). A detailed input (current intensity)/ output (action potential [AP] frequency) curve in response to a depolarizing current step was drawn for both groups of mice (Figure 5C). Pyramidal neurons infected with shLamb1 displayed a significant augmentation in firing frequency, as characterized by a leftward and upward shift of the inputoutput (I-O) curve over scrambled shRNA (Figure 5C). Meanwhile, knockdown of ACC LAMB1 produced a lowered rheobase in pyra- midal neurons (Figure 5D). The other AP parameters such as the AP threshold, amplitude, as well as half-width values were largely unaltered by knockdown of ACC LAMB1 (Supplemental Figure 7, A-D). Furthermore, we observed enhanced synaptic transmission in ACC pyramidal neurons derived from mice expressing shLamb1 in comparison with those from mice expressing scrambled shRNA (Figure 5, E and F). AMPA receptor-mediated evoked excitatory postsynaptic currents (AMPAR-eEPSCs) in pyramidal neurons of layers II and III in the ACC at a holding potential of $-70 \mathrm{mV}$ were recorded by applying local stimulation in layers V and VI in the presence of the inhibitory synaptic transmission antagonist picrotoxin $(100 \mu \mathrm{M})$ and the NMDA receptor antagonist AP5 $(50 \mu \mathrm{M})$. The amplitude of AMPAR-eEPSCs was much higher in the pyrami- 
A

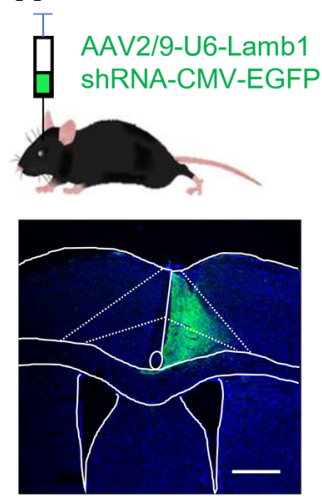

B

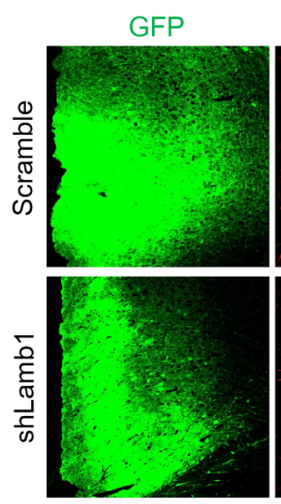

LAMB1

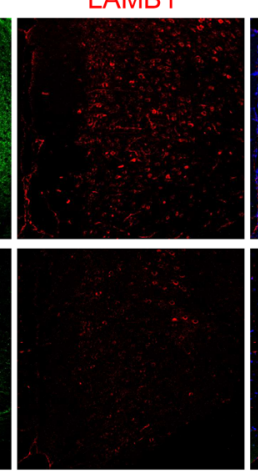

Merge

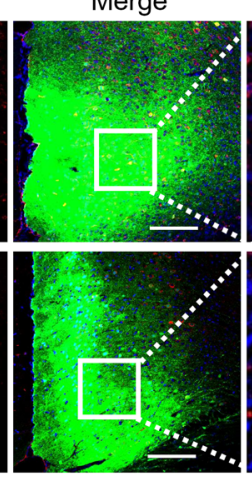

C

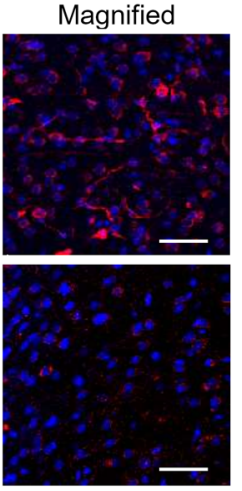

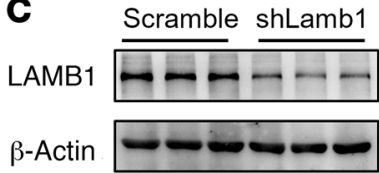

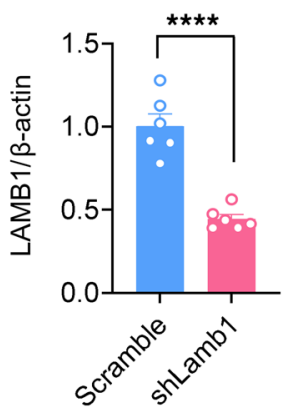

Ipsilateral

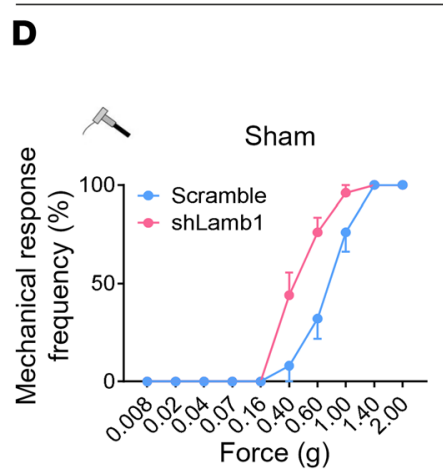

G

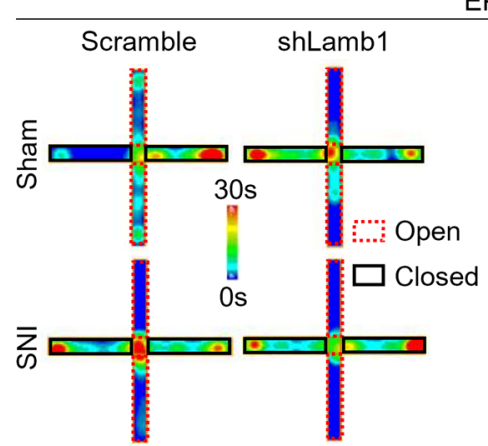

E

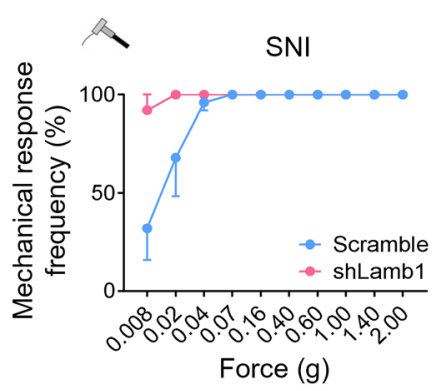

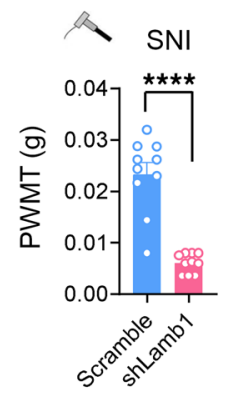

$\mathbf{F}$

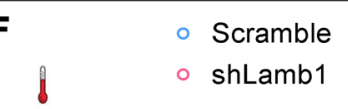

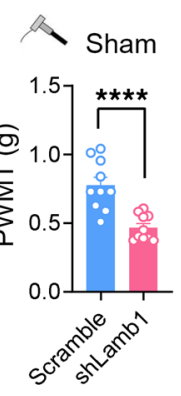

EPM
H

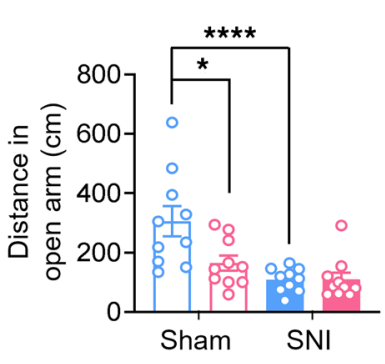

TST

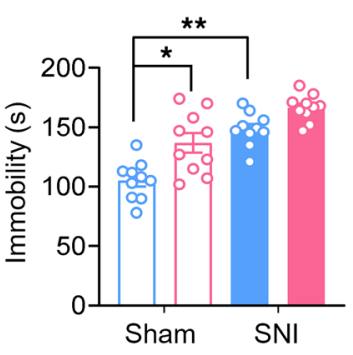

I

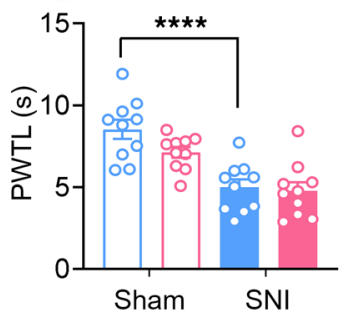

Figure 3. LAMB1 knockdown in the ACC induces pain hypersensitivity and anxiodepression. (A) Schematic diagram of intra-ACC virus injection into C57BL/6 mice $(n=10)$. Scale bar: $500 \mu \mathrm{m}$. (B and C) Double-immunofluorescence images (B) and Western blots (C) showing efficient LAMB1 knockdown in the ACC $(n=6) .{ }^{* * *} P<0.0001$, by 2 -tailed, unpaired separate variance estimation $t$ test. Scale bars: $200 \mu \mathrm{m}$ and $70 \mu \mathrm{m}$ (enlarged insets). ( $\mathbf{D}$ and $\left.\mathbf{E}\right)$ Stimulus response curve and mechanical threshold showing that ACC LAMB1 knockdown exacerbated ipsilateral mechanical sensitivity in sham-treated (D) and SNI-treated $(\mathbf{E})$ mice $(n=10)$. ${ }^{* * *} P<0.0001$, by Mann-Whitney $U$ test. $(\mathbf{F})$ Ipsilateral thermal sensitivity was unaltered by LAMB1 knockdown in the sham- or SNI-treated state $(n=10)$. ${ }^{* * *} P<0.0001$, by 1-way ANOVA with Tukey's multiple-comparison test. (G) Traveling trajectory in the EPM and quantitative summary showed that sham-treated mice expressing shLamb1 traveled shorter distances in the open arm ( $n=10$ ). ${ }^{*} P<0.05$ and ${ }^{* * * *} P$ $<0.0001$, by Kruskal-Wallis $H$ test with Nemenyi's multiple-comparison test. $(\mathbf{H})$ The TST showed that expression of AAV-shLamb1 resulted in longer immobility for the sham-treated mice and further exacerbated immobility following SNI $(n=10)$. ${ }^{*} P<0.05$ and ${ }^{* *} P<0.01$, by Kruskal-Wallis $H$ test with Nemenyi's multiple-comparison test. (I) The SPT showed a strong reduction in sucrose preference in shLamb1-expressing mice $(n=10)$. ${ }^{*} P<0.01$ and ${ }^{* * *} P<0.0001$, by 1-way ANOVA with Tukey's multiple-comparison test. Data are presented as the mean \pm SEM. See Supplemental Table 2 for detailed statistical information. PWMT, paw withdrawal mechanical threshold; PWTL, paw withdrawal thermal latency.

dal neurons of mice infected with shLamb1 than in those infected with control RNA (Figure 5F). Likewise, the AMPAR/NMDAR (NMDAR-mediated eEPSCs) ratio was higher in ACC pyramidal neurons after LAMB1 knockdown (Figure 5, G and H). In contrast, the amplitude of NMDAR-eEPSCs was not different between the
2 groups (Figure 5, G and H). To determine whether a presynaptic or postsynaptic mechanism contributes to the observed changes of synaptic function, we first focused on analyzing the pairedpulse ratio (PPR), which represents a short-lasting increase or decrease in the second eEPSC when it occurs shortly after the first 
A

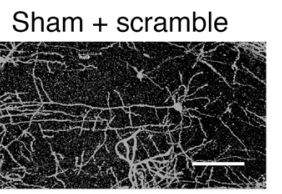

Sham + shLamb1

$\mathrm{SNI}+$ scramble

$\mathrm{SNI}+$ shLamb1
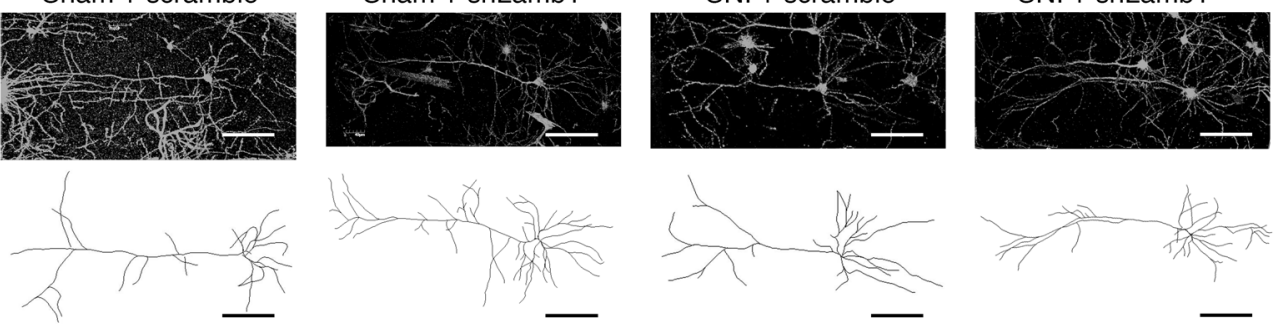

B
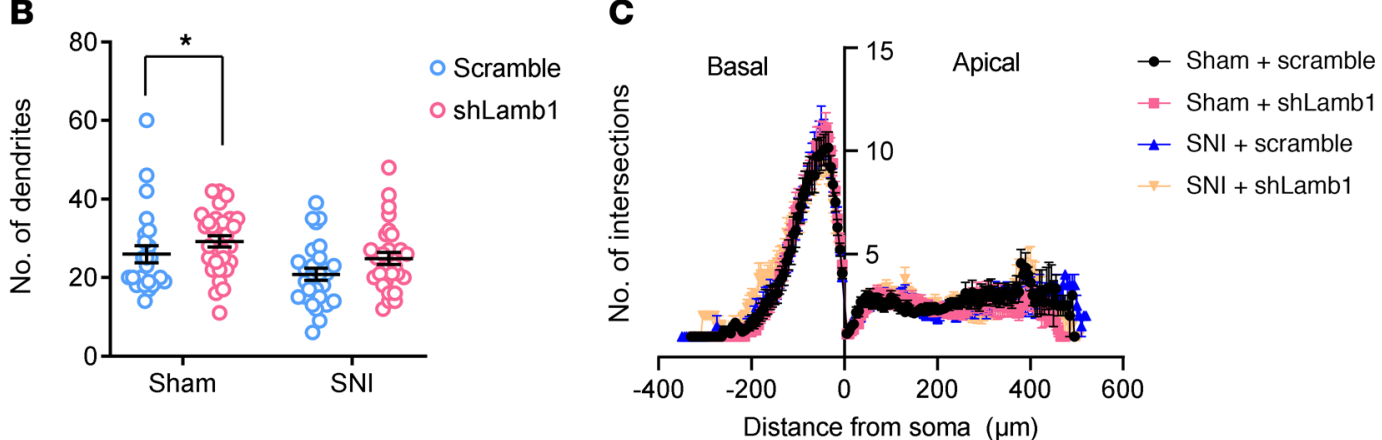

D
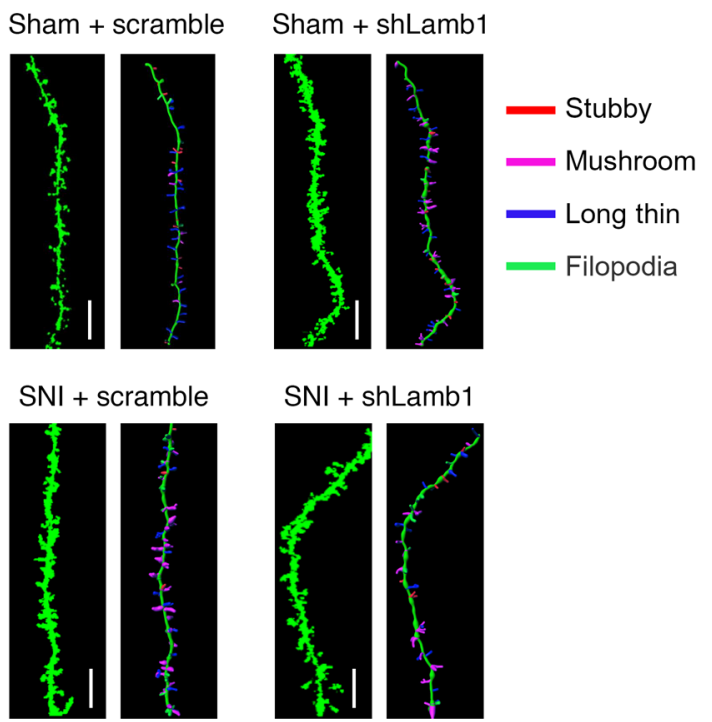

SNI + shLamb1

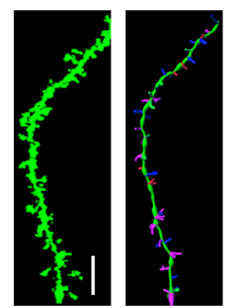

E
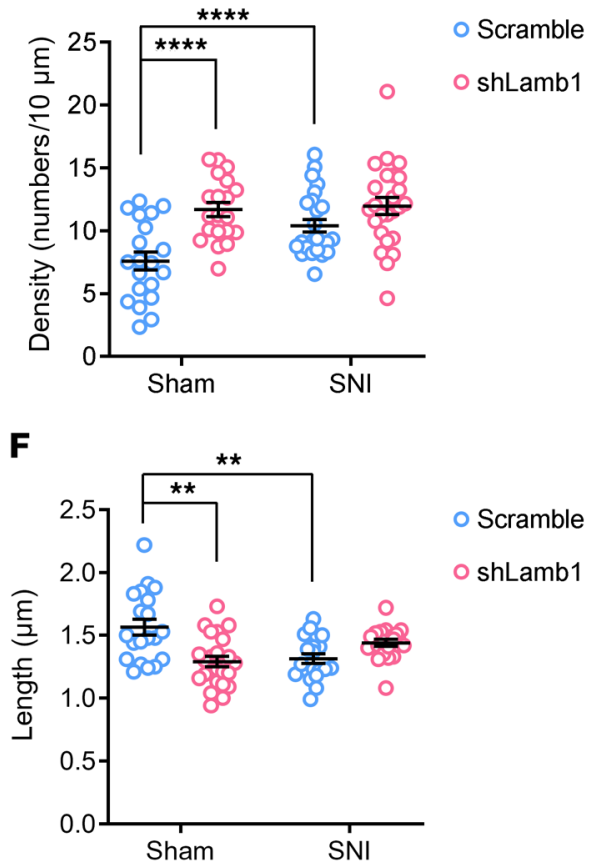

G Mushroom

Long thin

Filopodia

- Scramble o shLamb1
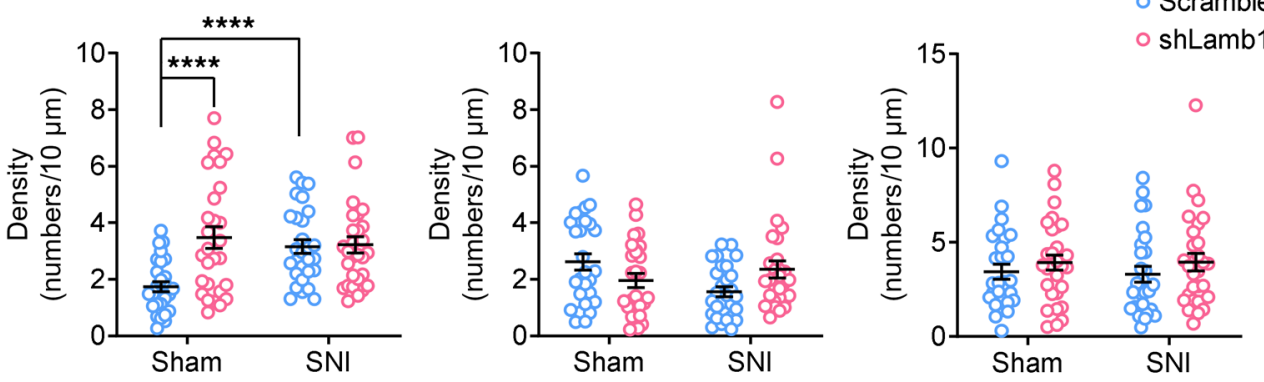
Figure 4. LAMB1 deficiency in the ACC induces apical dendritic spine remodeling of ACC pyramidal neurons. (A) Representative images of pyramidal neurons in the ACC derived from sham- and SNI-treated mice expressing scrambled shRNA or shLamb1 $(n=24-30)$. Scale bars: $100 \mu \mathrm{m}$. (B and C) Sholl analysis of dendritic branching complexity in the basal and apical dendrites of sham- or SNI-operated mice expressing scrambled shRNA or shLamb1 $(n=24-30)$. ${ }^{*} P<0.05$, by Kruskal-Wallis $H$ test with Nemenyi's multiple-comparison test. (D) Representative confocal stack and $3 \mathrm{D}$ reconstruction images of apical dendrites of ACC pyramidal neurons obtained from mice expressing shLamb1 or scrambled shRNA in both sham and SNI conditions ( $n=20-25)$. Scale bars: $5 \mu \mathrm{m}$. (E and F) Summary of spine density (E) and length (F) of apical dendrites of ACC pyramidal neurons obtained from mice expressing shLamb1 or scrambled shRNA in both sham and SNI conditions $(n=20-25) .{ }^{* *} P<0.01$ and ${ }^{* * *} P<0.0001$, by Kruskal-Wallis $H$ test with Nemenyi's multiple-comparison test. (G) Summary of the density of stubby-, mushroom-, long/thin-, and filopodiashaped spines on apical dendrites of ACC pyramidal neurons from mice of the above 4 groups $(n=22-29)$. ${ }^{* * *} P<0.0001$, by Kruskal-Wallis $H$ test with Nemenyi's multiple-comparison test. Data are presented as the mean \pm SEM. See Supplemental Table 2 for detailed statistical information.

and is well accepted as an indication of presynaptic mechanisms of long-term potentiation in the hippocampus (31). Paired-pulse facilitation (PPF) or paired-pulse depression (PPD) was observed in $84.2 \%$ and $15.8 \%$ of ACC pyramidal neurons, respectively. The average PPR was significantly reduced upon knockdown of LAMB1 (Figure 6, A and B), indicative of an increase in the transmitter release probability via a presynaptic mechanism. This presynaptic involvement was further consolidated by an increase in miniature EPSC (mEPSC) frequencies after knockdown of ACC LAMB1 (Figure 6, C and D). Meanwhile, the amplitude of mEPSCs was potentiated after loss of LAMB1, suggesting the involvement of a postsynaptic mechanism as well (Figure 6, C and E). The possible postsynaptic mechanism was further indicated by our observation of the effect of LAMB1 on the responsiveness of postsynaptic AMPARs. As shown in Figure 6, F and G, the AMPA-induced $(50 \mu \mathrm{M})$ current in pyramidal neurons was significantly enhanced by knockdown of LAMB1, whereas the NMDA-induced $(50 \mu \mathrm{M})$ current was not (Figure 6, F and G). Overall, these results suggest that LAMB1 deficiency potentiates synaptic transmission via both presynaptic and postsynaptic mechanisms that likely involve an increase in presynaptic transmitter release probability and responsiveness of postsynaptic AMPARs. In sum, we can infer from the above observations that LAMB1 deficiency in the ACC orchestrates structural and functional plasticity of pyramidal neurons.

LAMB1-knockdown mice exhibit altered Src/RhoA/LIMK/cofilin signaling and dysregulated F-actin in the ACC. We next asked the question: By which mechanism does ACC LAMB1 regulate dendritic spine remodeling? Rearrangement of the actin cytoskeleton in neurons has been linked to spine morphology and function and may also be involved in many pathophysiological processes (32, 33). The polymerized filamentous actin (F-actin) has also been shown to be increased in primary sensory neurons upon peripheral inflammation, and disruption of F-actin relieved the inflammatory pain (34). We thus asked whether LAMB1 possesses the ability to regulate actin dynamics in the ACC. F-actin labeling with phalloidin in both in vivo ACC tissue and in vitro cultured cortical neurons revealed significant enhancement in neurons expressing shLamb1 compared with those expressing scrambled shRNA (Fig- ure 7, A and B). These data indicate that the absence of LAMB1 resulted in abnormal actin organization at dendritic spines, raising the question of which events link activity-dependent changes in LAMB1 expression and actin disorganization. Integrins were first described as mediators of cellular adhesion, linking the cytoskeleton and the ECM (35). To determine whether - and with which integrin subunit - LAMB1 interacts in the ACC, we performed an immunoprecipitation assay with total ACC cell lysates. As shown in Figure 7C, LAMB1 strongly interacted with integrin $\beta 1$, and disruption of LAMB1 largely eliminated this interaction (Figure 7C). The laminin-integrin system has been shown to activate Src family of kinases in the development of oligodendrocytes (36), and Src kinase is a known upstream regulator of actin cytoskeleton (37). We then asked whether Src activity was decreased in the ACC of LAMB1-knockdown mice. We performed Western blot analysis with antibodies against the phosphorylated Tyr416 of Src, which recognizes the activated protein, and observed a significant reduction Src phosphorylation ( $\mathrm{p}-\mathrm{Src}$ ) after knockdown of LAMB1 in the ACC (Figure 7D). This result suggests that the absence of LAMB1 resulted in a Src activity-mediated actin reorganization in dendritic spines of the ACC.

It has been shown that Src could regulate actin organization through the small GTPase RhoA (38), which is a known regulator of cofilin activity and of dendritic spine actin organization (39, 40). Indeed activated RhoA, through the ROCK/LIMK pathway, has been shown to inhibit cofilin by inducing its phosphorylation on Ser3, thereby triggering proper spine remodeling (41-43). In addition, activated Src can phosphorylate the RhoA-specific GTPase-activating protein (GAP) p190RhoGap, thereby inducing its GAP activity and decreasing the levels of GTP-RhoA (44-46). Consistent with this potential mechanism in other systems, we observed that lysates of ACC tissue extracted from LAMB1-knockdown mice had decreased levels of tyrosine-phosphorylated p190RhoGAP and increased levels of active RhoA, as assayed by immunoprecipitation and Western blotting (Figure 7, $\mathrm{E}$ and $\mathrm{F}$ ). Given the increased RhoA activity in the LAMB1-knockdown mice, levels of downstream effectors of the RhoA signaling cascade, such as ROCK2 and LIMK2, but not LIMK1, were significantly enhanced in the ACC (Figure 7G). A key downstream target of ROCK/LIMK signaling is cofilin, the major actin depolymerizing factor, which is inactivated by phosphorylation at Ser3 (47). LAMB1-knockdown mice had unaltered total cofilin levels, but significantly increased levels of the inactive form of cofilin (p-cofilin; Figure 7G), indicating that levels of the active form of cofilin declined after LAMB1 knockdown in the ACC. Altogether, these results suggest that spine remodeling elicited by LAMB1 loss in the ACC may be caused by Src-dependent activation of the RhoA/ROCK/LIMK signaling cascade, leading to abnormally decreased levels of active cofilin and eventually resulting in actin rearrangement.

LAMB1 knockdown leads to increased AMPAR membrane trafficking and thus results in increased ACC pyramidal neuron activity. Given the importance of RhoA signaling and actin stability in AMPAR membrane trafficking (48-51), together with our observation of enhanced AMPAR-eEPSCs in LAMB1-knockdown mice, we went on to determine whether LAMB1 knockdown leads to changes in trafficking of AMPARs to the plasma membrane. To 
A

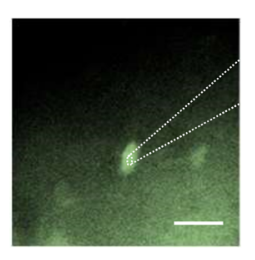

B

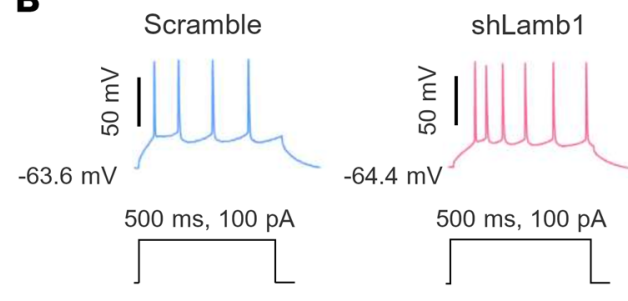

C

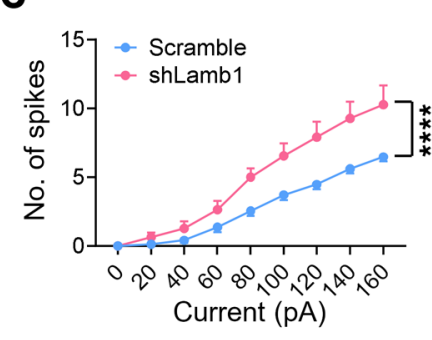

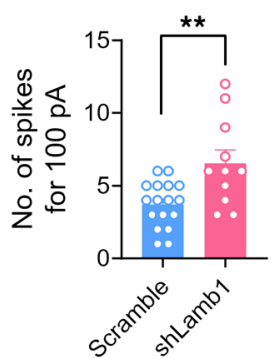

D

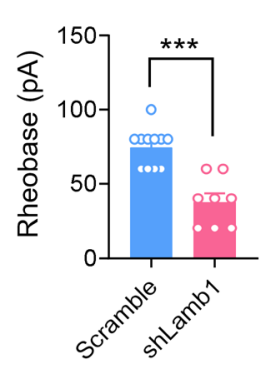

G

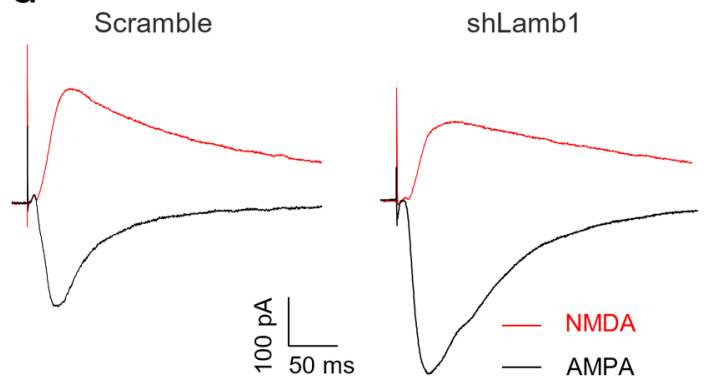

E

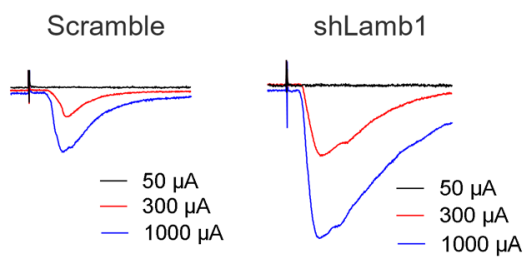

$\mathbf{F}$
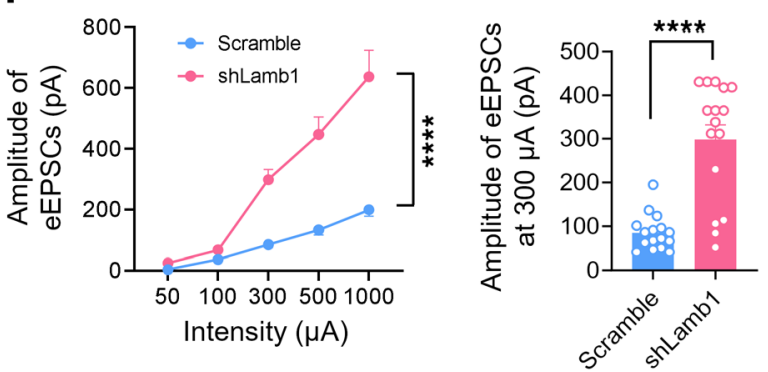

H

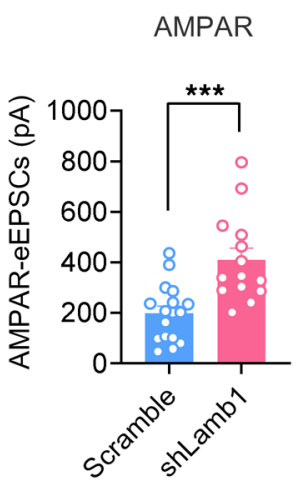

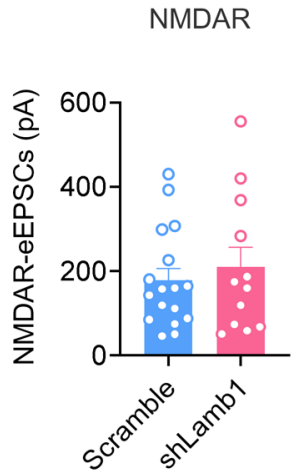

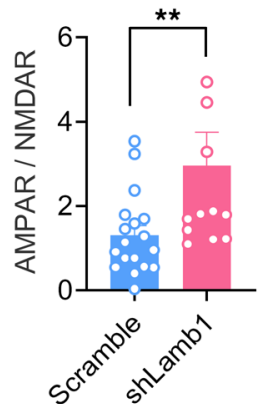

Figure 5. LAMB1 deficiency evokes neuronal hyperexcitability and synaptic potentiation in ACC pyramidal neurons. (A) Whole-cell patch-clamp recording from ACC layer II/III pyramidal neurons. Scale bar: $50 \mu \mathrm{m}$. (B) APs induced by current injection at 100 pA in neurons expressing shLamb1 or scrambled shRNA ( $n=11-17)$. (C) Left: I-O curve in response to a depolarizing current step (20 pA step, 500 ms duration) showing a higher firing frequency in mice expressing shLamb1 $(n=11-17)$. ${ }^{* * *} P<0.0001$, by Friedman's $M$ test. Right: Typical result at an intensity of 100 pA. ${ }^{* *} P<0.01$, by 2 -tailed, unpaired separate variance estimation $t$ test. (D) A lowered rheobase was observed after LAMB1 knockdown $(n=8-11)$. ${ }^{* * *} P<0.001$, by Mann-Whitney $U$ test. (E and $\mathbf{F}$ ) Representative traces (E) and I-O curve (F) of AMPAR-mediated eEPSCs following stimulation of layer V/VI ACC pyramidal neurons in mice of both genotypes $(n=16) .{ }^{* * *} P<0.0001$, by Friedman's $M$ test (left panel) and Mann-Whitney $U$ test (right panel). Right panel in F shows typical quantification of AMPARs-eEPSCs evoked by $300 \mu$ A stimulation. (G and $\mathbf{H}$ ) Representative traces (G) and quantitative summary (H) of AMPAR/NMDAR EPSC ratios for mice of both genotypes $(n=12-18) .{ }^{* *} P<0.001$, by 2 -tailed, unpaired $t$ test for AMPARs-eEPSCs; ${ }^{* *} P<0.01$, by Mann-Whitney $U$ test for NMDAR-eEPSCs and AMPAR/NMDAR. Data are presented as the mean \pm SEM. See Supplemental Table 2 for detailed statistical information.

test this, we compared subcellular distribution of different glutamate receptor subunits in the ACC in mice expressing scrambled shRNA or shLamb1. As shown in Figure 8, A and B, the AMPAR subunit GluR1 in the membrane fraction of ACC tissues was significantly enhanced in LAMB1-knockdown mice, with no significant difference observed for GluR2 subunit (Figure 8, A and B). This is consistent with the observation of increased GluR1 insertion into the plasma membrane during synaptic plasticity in different regions $(42,52-58)$. In addition to AMPARs, the NMDA receptor (NMDAR) subunit NR2A showed enhanced membrane trafficking as well after LAMB1 knockdown, whereas the membrane NR1 and NR2B subunits were largely unaltered (Figure 8, A and B). Moreover, we observed prominent upregulation of postsynaptic density
95 (PSD95) expression in the plasma membrane of the ACC in mice expressing shLamb1 compared with those expressing control RNA (Figure 8, A and B). We also confirmed increased PSD95 expression in cultured cortical neurons expressing shLamb1 (Figure 8C). Together, this increased amount of AMPARs and NMDARs in the plasma membrane suggested the enhancement of AMPAR and NMDAR delivery to the plasma membrane of postsynaptic densities (PSDs) after knockdown of LAMB1.

To better understand the in vivo functional consequences of enhanced AMPAR and NMDAR trafficking to the membrane in LAMB1-knockdown mice, we used fiber photometry to record the activity of GCaMP6s-expressing pyramidal neurons in the ACC during tail suspension by unilateral intracingulate injection of an 
A

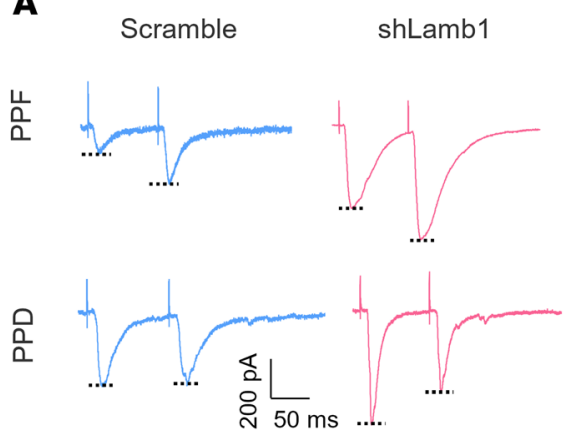

B

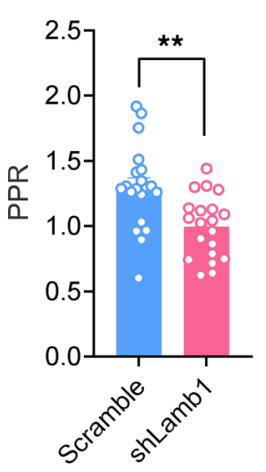

$\mathbf{F}$
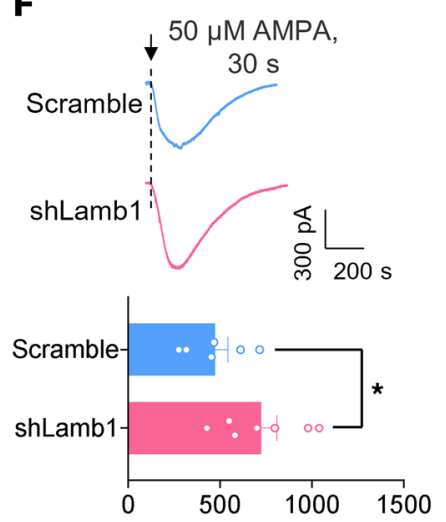

AMPA-induced current (pA)
G
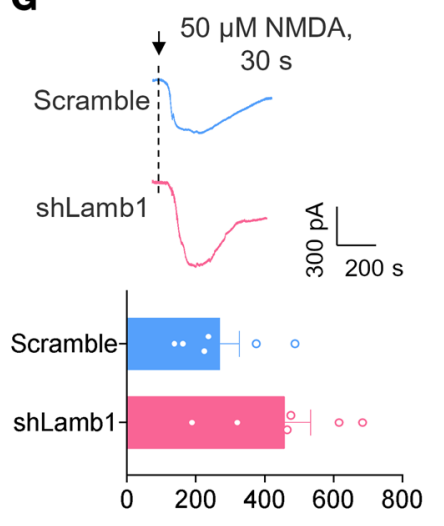

NMDA-induced current (pA)
C

Scramble

shLamb1

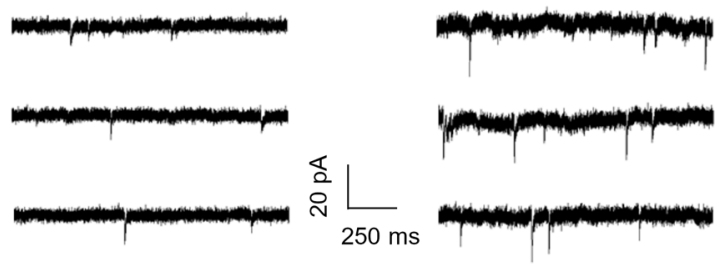

D
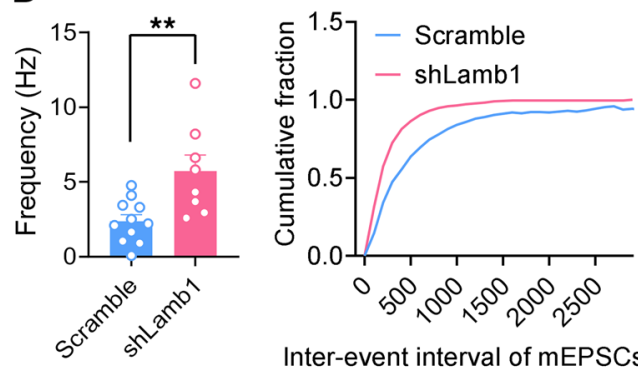

Inter-event interval of mEPSCs (ms)

E

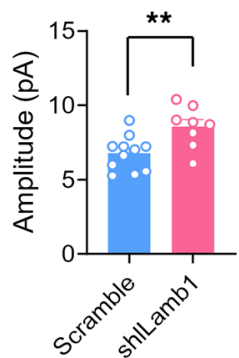

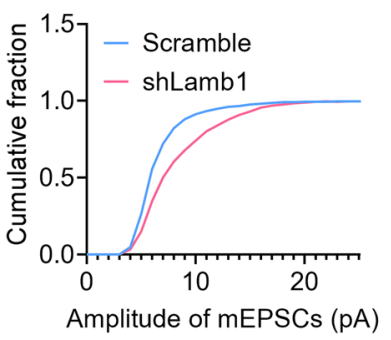

Figure 6. Both presynaptic and postsynaptic mechanisms are involved in the synaptic potentiation induced by LAMB1 knockdown in the ACC. Representative paired-pulse traces (A) and quantitative summary (B) of PPF or PPD showing that LAMB1 knockdown led to a reduced PPR $(n=20)$. ${ }^{* *} P<0.01$, by 2 -tailed, unpaired $t$ test. (C-E) Representative traces (C) and quantification of mEPSC frequency (D) and amplitude (E) showing that LAMB1 knockdown significantly increased mEPSC frequency and amplitude $(n=8-11)$. ${ }^{*} P<0.01$, by 2-tailed, unpaired separate variance estimation $t$ test (D) and 2-tailed, unpaired $t$ test $(\mathbf{E})$. (F and $\mathbf{G})$ Inward currents induced by bath-applied AMPA $(50 \mu \mathrm{M})(\mathbf{F})$ or NMDA $(50 \mu \mathrm{M})(\mathbf{G})$ in mice of both genotypes $(n=6-7)$. ${ }^{*} P<0.05$, by 2-tailed, unpaired $t$ test. Data are presented as the mean \pm SEM. See Supplemental Table 2 for detailed statistical information.

AAV vector containing CaMKII-GCaMP6s (experimental schematic paradigm is shown in Figure 8, D and E). Pain hypersensitivity and prolonged immobility were observed in mice expressing shLamb1, as compared with those expressing scrambled shRNA (Figure 8, F and G). During tail suspension, we noted that ACC activity in shLamb1-expressing mice was significantly elevated, as characterized by larger calcium transients than those elicited in mice expressing control RNA (Figure 8, $\mathrm{H}-\mathrm{J}$ ). These data indicate that the pain hypersensitivity and depressive behavior observed in LAMB1-knockdown mice were associated with increased ACC neuronal activity.

Activation of the LAMB1-integrin $\beta 1$ system relieves the established pain hypersensitivity and psychiatric disorders after SNI. Finally, we asked whether ACC LAMB1 has therapeutic effects on neuropathic pain and aversive emotion. Since the Lamb1 gene is too large to be packaged into the AAV for overexpression, we turned to the agonist of integrin $\beta 1$ with which LAMB1 mainly interacts. We found that bilateral ACC injection of the integrin $\beta 1$ activator pyrintegrin $(1 \mathrm{mM}, 600 \mathrm{~nL})$ did not alter the basal nociception of mechanical or thermal stimuli (Figure 9A). In contrast, delivery of pyrintegrin into the ACC significantly relieved the ipsilateral mechanical allodynia and thermal hyperalgesia induced by SNI, as compared with vehicle delivery, with no significant effect on contralateral hyperalgesia (Figure 9, B-D, and Supplemental Figure 8, A-C). In addition, in SNI-operated mice, intra-ACC administration of pyrintegrin increased open-arm exploration in the EPM without affecting total distance, decreased the immobility in the TST, and increased sucrose preference (Figure 9, E-G, and Supplemental Figure 8, D and $\mathrm{E}$ ), which are findings indicative of its desirable anxiolytic and antidepressive effects in the neuropathic state.

We then addressed the question of whether pyrintegrin alleviates neuropathic pain and its related aversion via the Src/cofilin signaling pathway. Western blot analysis revealed that SNI induced a significant decrease in $\mathrm{p}-\mathrm{Src}$, a significant increase in p-cofilin, as well as significant upregulation of PSD95 in the ACC, similar to what we observed in LAMB1-knockdown mice (Figure $10, A-C)$. Importantly, intra-ACC injection of pyrintegrin reversed these effects (Figure 10, A-C). Moreover, enhancement of F-actin 
A
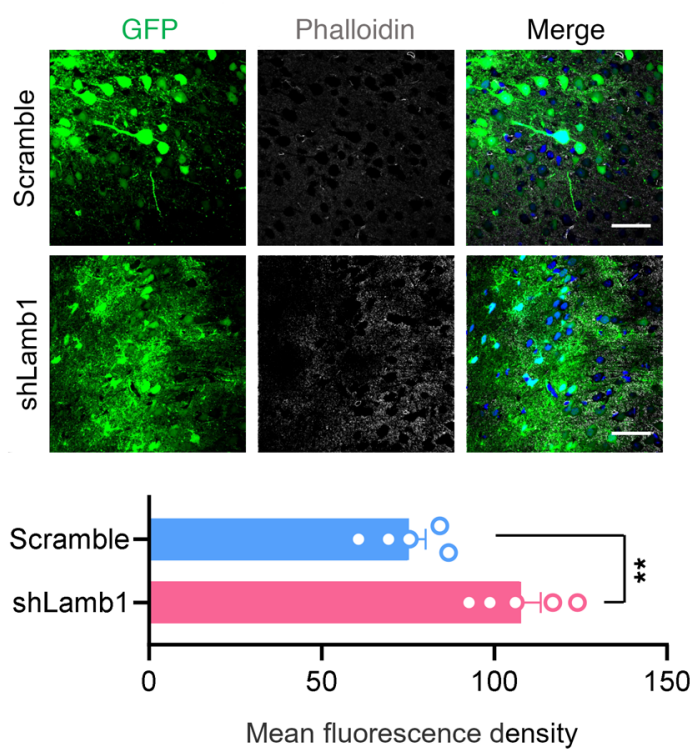

C
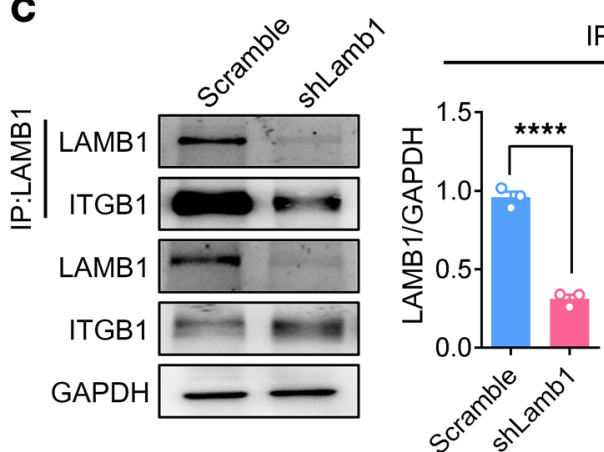

P:LAMB1

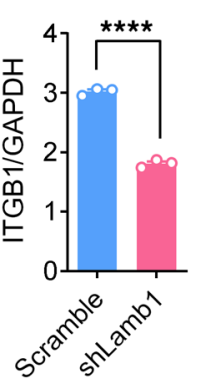

E
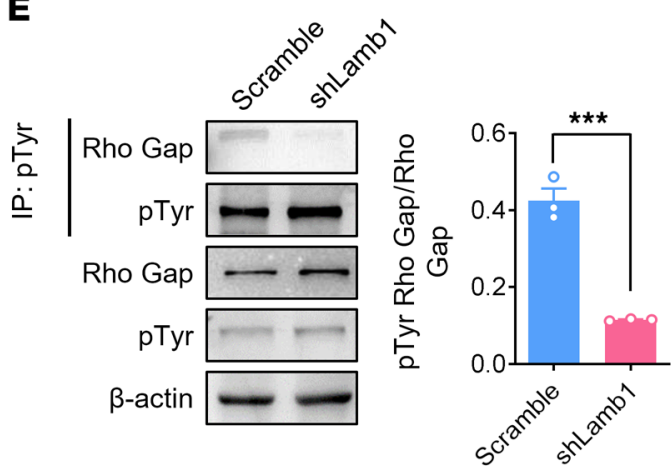

G

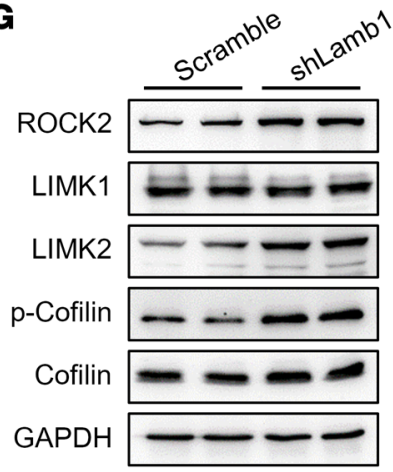

Merge
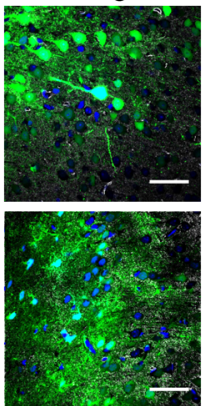

50

(1)

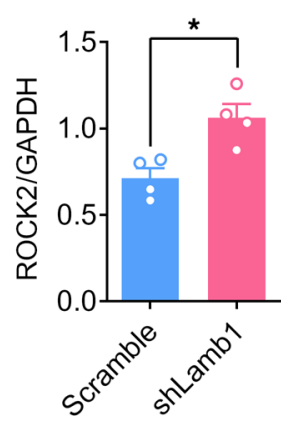

B
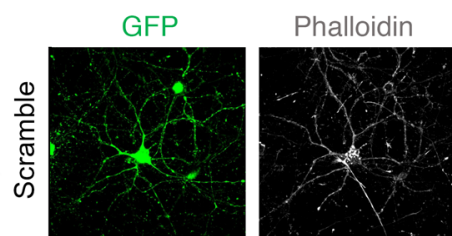

Merge
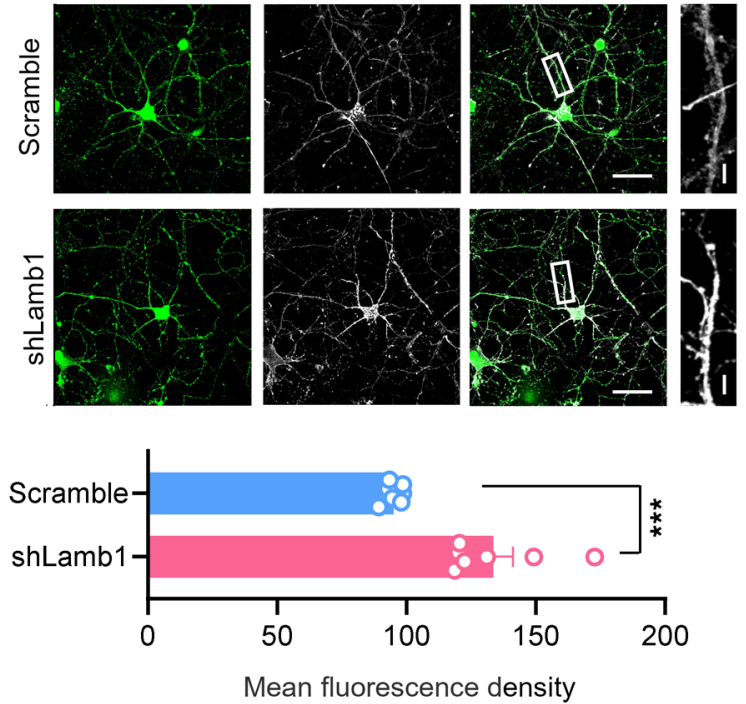

D

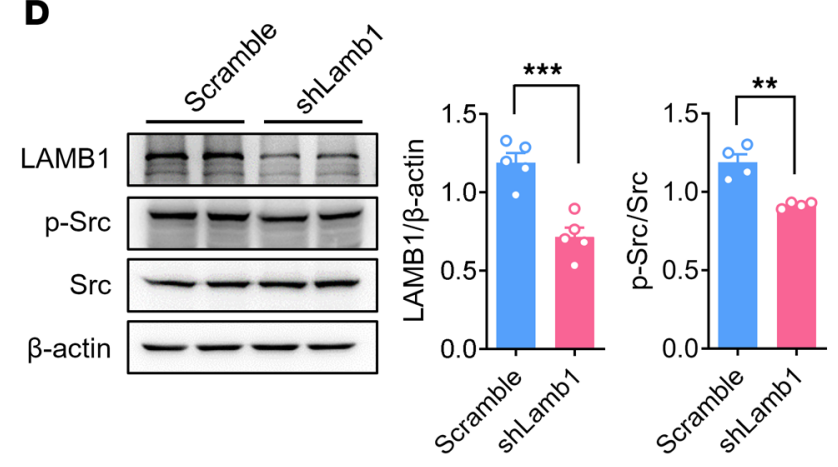

$\mathbf{F}$

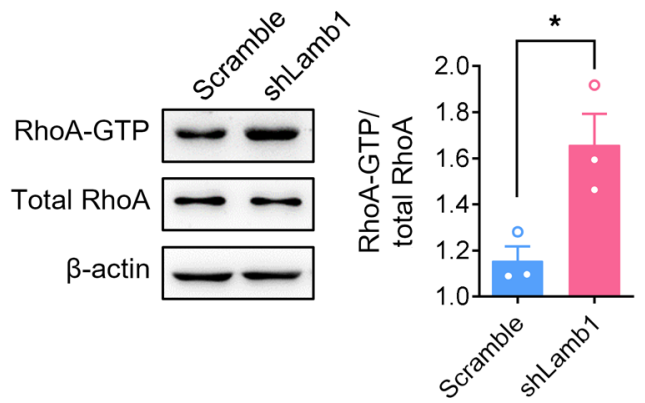

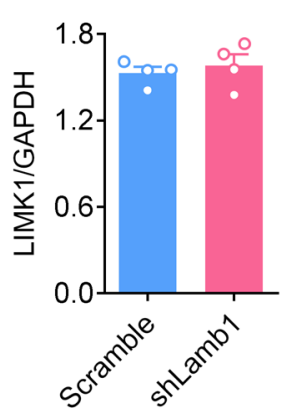
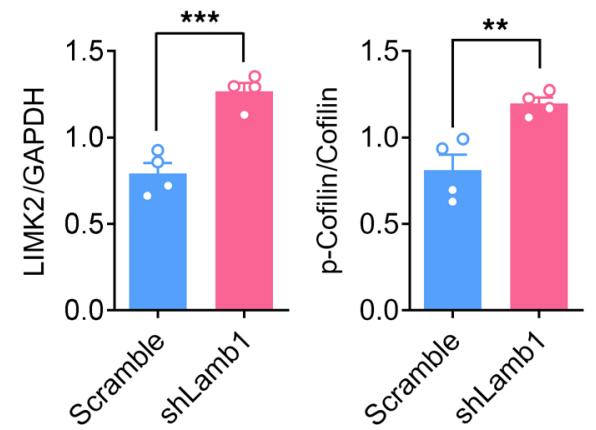
Figure 7. LAMB1-knockdown mice exhibit altered Src/RhoA/LIMK/cofilin signaling and dysregulated F-actin in the ACC. (A and B) Confocal images of F-actin staining with phalloidin in ACC slices from mice expressing scrambled shRNA or shLamb1 $(\mathbf{A})(n=5)$ and cultured cortical neurons transfected with shLamb1 or scrambled shRNA $(\mathbf{B})(n=7) .{ }^{* *} P<0.01$ and ${ }^{* * *} P<0.001$, by 2 -tailed, unpaired $t$ test $(\mathbf{A})$ and Mann-Whitney $U$ test (B). Scale bars: $30 \mu \mathrm{m}$ (A); $50 \mu \mathrm{m}$ and $5 \mu \mathrm{m}$ (enlarged insets)(B). (C) Representative immunoblots and quantitative summary of immunoprecipitated LAMB1 and integrin $\beta 1$ in ACC lysates derived from mice expressing scrambled shRNA or shLamb1 $(n=3) .{ }^{* * *} P<0.0001$, by 2-tailed, unpaired $t$ test. (D) Representative immunoblots and quantitative summary of levels of LAMB1, p-Src, and total Src in ACC lysates from mice expressing scrambled shRNA or shLamb1 $(n=4-5)$. ${ }^{*} P<0.01$ and ${ }^{* *} P<0.001$, by 2 -tailed, unpaired $t$ test (LAMB1/ $\beta$-actin) and 2 -tailed, unpaired separate variance estimation $t$ test ( $\mathrm{p}-\mathrm{Src} / \mathrm{Src}$ ). (E) Representative immunoblots of immunoprecipitated $p$-Tyr and quantitative summary of $p$-Tyr, p190RhoGAP, and $\beta$-actin (loading control) levels $(n=3)$. ${ }^{* *} P<0.001$, by 2 -tailed, unpaired separate variance estimation $t$ test. (F) Representative immunoblots and quantitative summary of RhoA-CTP and total RhoA levels using a pulldown assay $(n=3)$. ${ }^{*} P<0.05$, by 2 -tailed, unpaired $t$ test. (G) Representative immunoblots and quantitative summary of ROCK2, LIMK1, LIMK2, $\mathrm{p}$-cofilin, and total cofilin levels in ACC lysates derived from mice expressing scrambled shRNA or shLamb1 $(n=4) .{ }^{*} P<0.05$, ${ }^{* *} P<0.01$, and ${ }^{* *} P<$ 0.001 , by 2 -tailed, unpaired $t$ test. Data are presented as the mean \pm SEM. See Supplemental Table 2 for detailed statistical information.

induced by LAMB1 knockdown in cultured cortical neurons was normalized by the presence of pyrintegrin $(1 \mu \mathrm{M})$ in the medium (Figure 10D). In further support of this observation, bath application of pyrintegrin $(20 \mu \mathrm{M})$ largely relieved the hyperexcitability of ACC neurons in SNI-operated mice, as characterized by the reduction of AP frequencies and the elevation of AP rheobase after pyrintegrin treatment (Figure 10E). Potentiated AMPARs-eEPSCs in the neuropathic state were significantly alleviated as well by pyrintegrin administration (Figure 10F). Furthermore, we confirmed the effect of pyrintegrin in the above experiments by knocking down integrin $\beta 1$. As shown in Supplemental Figure $8, \mathrm{~F}-\mathrm{H}$, knockdown of integrin $\beta 1$ in the ACC led to a significant reduction in p-Src and a significant increase in p-cofilin (Supplemental Figure 8, F-H). Supplementation of pyrintegrin in the ACC eliminated these changes (Supplemental Figure 8, F-H).

Last, we tested whether ACC LAMB1 is involved in depression without chronic pain. We used 2 rodent models of depression, one involving chronic exposure to corticosterone (CORT) and the other involving chronic restraint stress (CRS). Mice exposed to chronic CORT or CRS displayed anxiety- and depressionrelated behaviors, including decreased center area traveling in the open field test (OFT), reduced open-arm exploration in the EPM, increased immobility in the TST, and decreased sucrose preference (Figure 11, A-D, Supplemental Figure 9, A and B, for CORT; Figure 11, F-I, and Supplemental Figure 9, C and D, for CRS). In contrast to the significant downregulation observed in mice with chronic pain-related aversion, we detected no significant changes in LAMB1 expression in the ACC of mice subjected to chronic CORT or CRS exposure (Figure 11, E and J). These results show the specific involvement of ACC LAMB1 in the development of chronic pain and associated depression but not in non-pain-related depression. Our assumption was further supported in another chronic inflammatory pain model by the downregulation of ACC LAMB1 induced by unilateral injection of CFA into the hind paw of mice (Supplemental Figure 10, A-G). Additionally, the changes in ACC LAMB1 levels following SNI were not sex specific, as female mice also showed reduced LAMB1 levels in the ACC after SNI (Supplemental Figure 11, A and B). Overall, we can conclude from the above observations that activation of ACC LAMB1/integrin $\beta 1$ signaling may represent a potential therapeutic target for the treatment of neuropathic pain and pain-related anxiodepression.

\section{Discussion}

The results of the present study led us to propose the model represented schematically in Figure 12. Peripheral nerve injury leads to downregulation of LAMB1 in the ACC. This reduced interaction with integrin $\beta 1$ brings about decreased phosphorylation of Src kinase, which causes a reduction in expression of the RhoAspecific GAP p190RhoGap, thereby increasing GTP-RhoA levels. Elevated RhoA levels activate ROCK2 and subsequently LIMK2, which phosphorylates cofilin and deactivates cofilin activity. This results in rearrangement of the actin cytoskeleton, which further leads to an increase in presynaptic transmitter release probability as well as abnormal postsynaptic spine remodeling and synaptic trafficking of AMPARs, and hence potentiates synaptic function. The resultant structural and functional plasticity collectively contributes to an exaggerated pain response and related anxiety and depression. Thus, this study primarily clarifies how LAMB1, a key ECM element, conveys extracellular alterations to intracellular structural and functional plasticity and modulates neuropathic pain and associated anxiodepression.

The most striking finding of this study was the identification of LAMB1, a key ECM element in the ACC, as a key determinant of neuropathic pain and anxiodepressive consequences. Despite mounting evidence for the role of the ACC in the generation of neuropathic pain and related aversion $(8-15,17)$, much attention has been paid to intracellular plasticity, but the extracellular changes have long been overlooked. A recent study demonstrated a sustained anatomical, physiological, and biochemical dysregulation of the hippocampal dentate gyrus ECM after peripheral neuropathy that was specifically characterized by decreased hippocampal dendritic complexity, an altered ECM microarchitecture, and decreased ECM rigidity (19). This plasticity in the hippocampal ECM was shown to be associated with pain and cognitive deficit characteristics in patients with pain (19). Apart from cognitive deficits, patients with neuropathic pain suffer more from psychiatric disorders, such as anxiety and depression (1-4). However, it remains unclear whether and which element of the ECM undergoes plastic changes in the ACC, a well-studied brain area responsible for sensory and emotional responses, and how it participates in the modulation of aversive emotion in neuropathic pain. Using RNA-Seq, we detected a total of 565 DEGs in the ACC, 56 days after SNI, and a significant enrichment of genes in various pathways, especially those for ECM-receptor interaction and focal adhesion. Among ECM-related pathway genes, we detected significant transcriptional downregulation in the ACC of the ECM element LAMB1, which we verified at both mRNA and protein levels. To further determine the causal relationship between plastic changes in ACC LAMB1 and neuropathic pain, we knocked down ACC LAMB1 and found that loss of LAMB1 in the ACC exacerbated pain sensitivity and induced anxiety and 
A

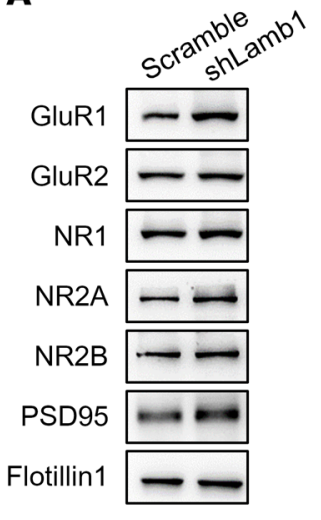

B

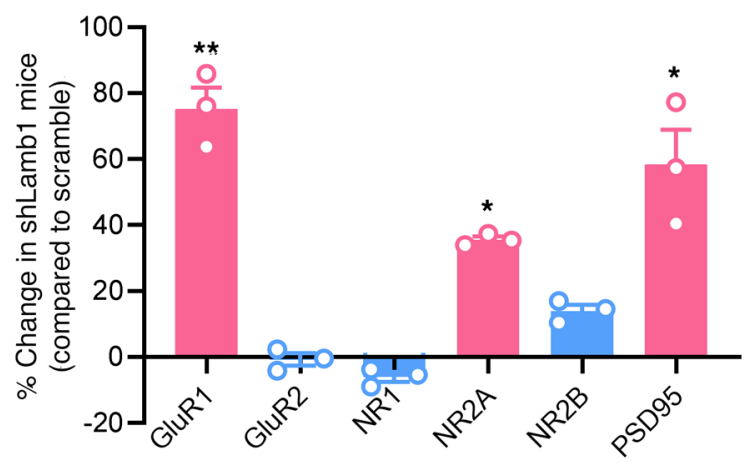

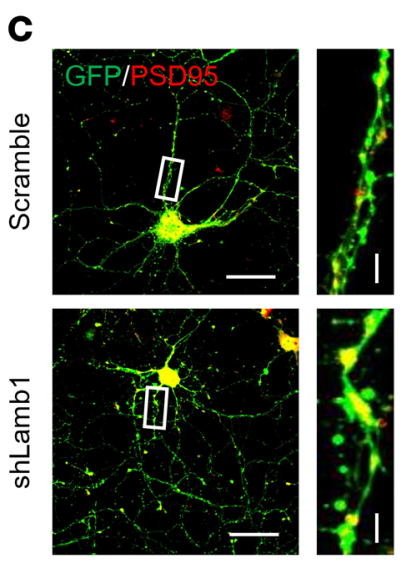

D

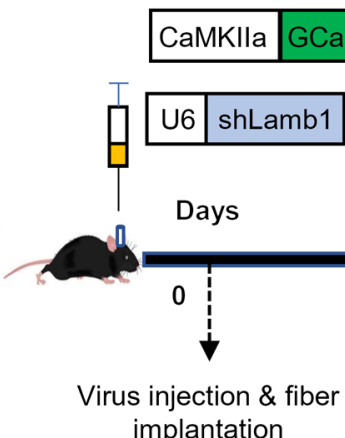

TST \& Fiber photometry \begin{tabular}{|l|l|}
\hline MV & mCherry \\
\hline
\end{tabular}

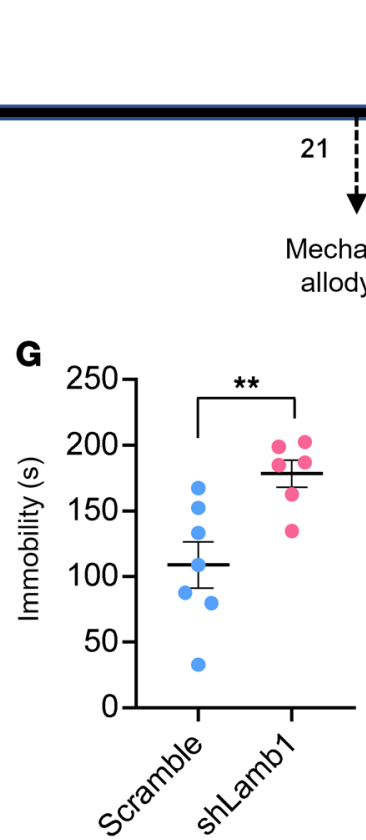

H
E
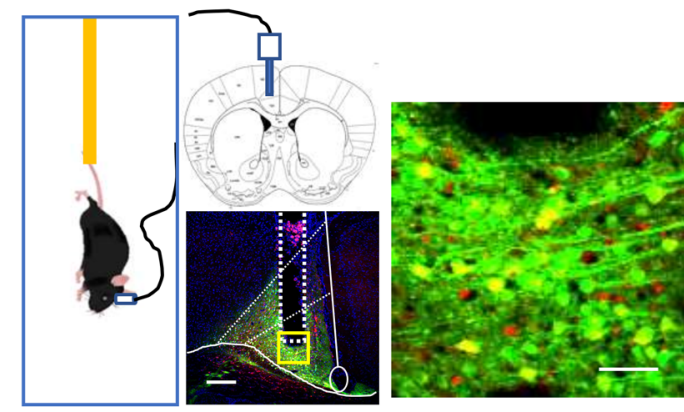

$\mathbf{F}$

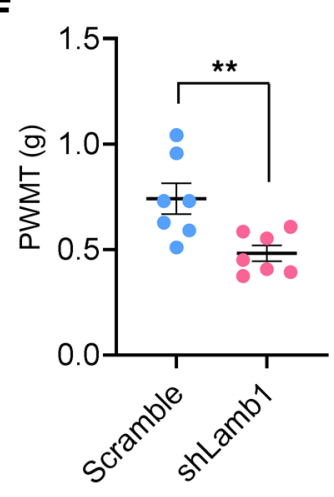

I

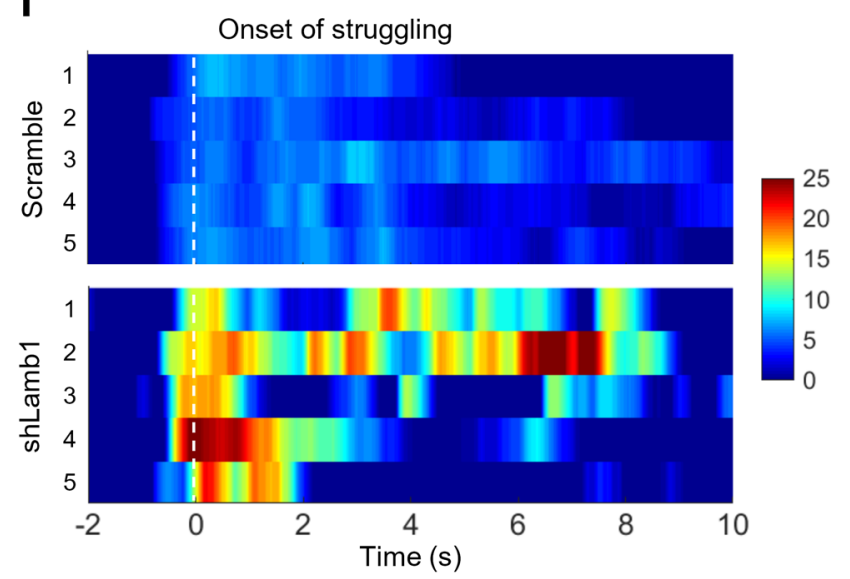

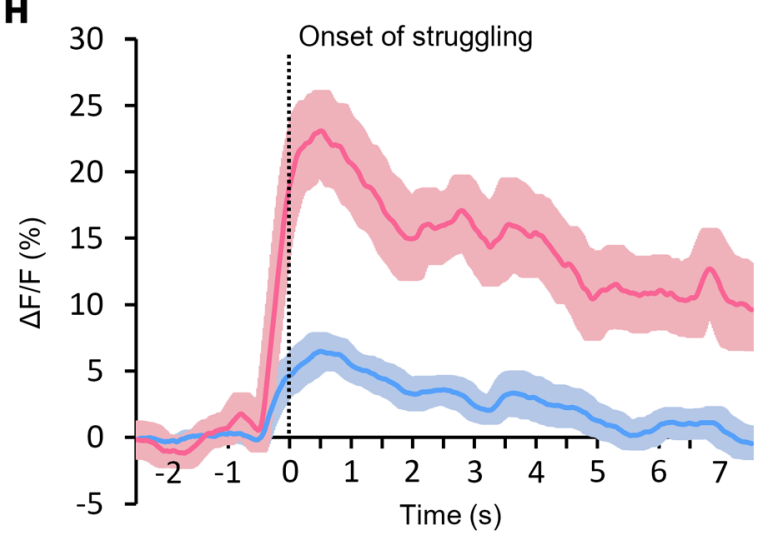

J

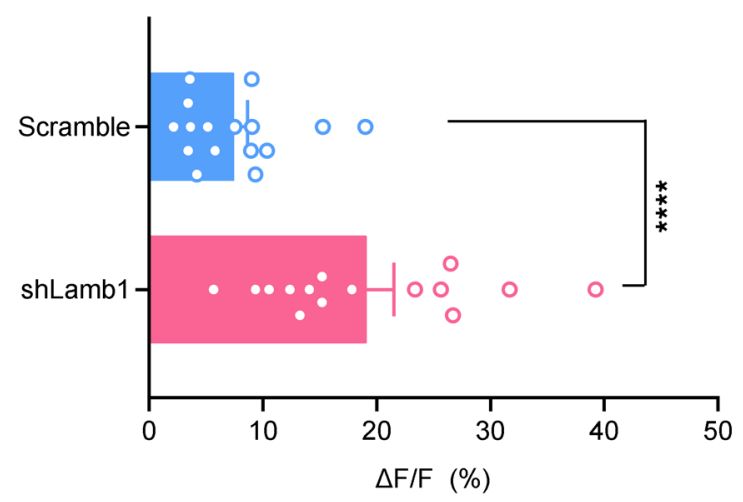


Figure 8. LAMB1 knockdown leads to increased AMPAR membrane trafficking and increased activity of ACC pyramidal neurons. ( $A$ and $B$ ) Representative immunoblots (A) and quantitative summary (B) showing the expression of AMPARs and NMDAR subunits in the membrane fraction of ACC tissue from mice expressing scrambled shRNA or shLamb1 $(n=3)$. For the quantitative analysis in B, a 2-step normalization was performed. Step 1: each blot was normalized (i.e., GluR1, GluR2, NR1, NR2A, NR2B, PSD95) to the loading control Flotillin 1 in the scrambled and shLamb1 groups, respectively; step 2: each subunit in the shLamb1 group was normalized to the scrambled group to assess the changes in each subunit. ${ }^{*} P$ $<0.05$ and ${ }^{* *} P<0.01$, by 2 -tailed, unpaired $t$ test. (C) Immunofluorescence images showing upregulated PSD95 expression in cultured cortical neurons expressing shLamb1 compared with expression of scrambled shRNA ( $n=$ 6). Scale bars: $50 \mu \mathrm{m}$ and $5 \mu \mathrm{m}$ (enlarged insets). ( $\mathbf{D}$ and $\mathbf{E})$ Experimental schematic diagram showing virus injection, optical fiber placement in ACC and behavioral test as well as fiber photometry recording during tail suspension test in mice expressing scrambled shRNA and shLamb1 $(n=6-7)$. Scale bars: $200 \mu \mathrm{m}$ and $30 \mu \mathrm{m}$ (enlarged insets) (E). (F and G) Mechanical threshold $(\mathbf{F})$ and immobility duration (C) in the tail suspension test in mice expressing scrambled shRNA or shLamb1 $(n=6-7)$. ${ }^{*} P<0.01$, by 2-tailed, unpaired $t$ test. (H-J) Representative photometric traces $(\mathbf{H})$ as shown in heatmaps (I) and quantitative summary (J) from 5 independent experiments of peak GCaMP6s signals locked to the onset of struggling. In the heatmaps I, each row in the $y$ axis represents GCaMP6s signals from 5 mice of each group. ${ }^{* * *} P<0.0001$, by Mann-Whitney $U$ test. Data are presented as the mean \pm SEM. See Supplemental Table 2 for detailed statistical information.

depression-like behaviors. These results suggest that LAMB1 in the ACC may negatively regulate neuropathic pain and anxiodepressive consequences.

In contrast, LAMB1 in the ACC may not be involved in depression with no pain, since we found no significant alteration of LAMB1 in the ACC in rodent models of chronic CORT and CRS exposure. In support of this assumption, previous studies have shown a significant decrease in laminin in the parieto-occipital cortex in patients with depression and that antidepressant treatment reverses this effect (25). Chronic stress decreases laminin levels in the frontal cortex and hippocampus (24). These data indicate that depression comorbid with neuropathic pain and depression without pain may involve laminin action in different brain regions. Additionally, elevated LAMB1 in the hippocampus and anterior temporal neocortex was shown to be associated with intractable epilepsy, and decreased LAMB1 expression in the hippocampus has been linked to spatial learning $(27,59,60)$.

Then how does LAMB1 in the ACC modulate neuropathic pain and related anxiodepressive consequences? Structural and functional plasticity in the ACC is assumed to serve as a cellular basis for neuropathic pain and related anxiodepression $(13,14$, 16, 17). ECM molecules, including LAMB1, have been linked to synapse stabilization, plasticity, and metaplasticity in several psychiatric disorders $(18,27-29)$. Another intriguing finding of this study entails the contribution of LAMB1 to the ACC in negatively orchestrating cingulate structural and functional plasticity. In the present study, we demonstrated that loss of LAMB1 produced prominent spine remodeling of ACC pyramidal neurons. This was supported by our observation of widespread expression of LAMB1 in $\mathrm{CaMKII}^{+}$neurons. These results confirm that LAMB1 contributes to the maintenance of synaptic spine stabilization in ACC pyramidal neurons and that disruption of LAMB1 induces abnor- mal spine remodeling. It is well accepted that functional synaptic plasticity is closely related to the remodeling of synaptic spines at a structural level and that this plasticity and remodeling both contribute to many physiological and pathological processes, such as chronic pain (61). In good agreement with spine remodeling, electrophysiological testing revealed that knockdown of LAMB1 drove ACC pyramidal neurons into a hyperexcitable state. Meanwhile, AMPAR-mediated synaptic transmission was significantly potentiated by silencing of LAMB1. Further mechanistic analysis revealed that both pre- and postsynaptic mechanisms were involved in the above-described synaptic modulation by LAMB1. Overall, these results suggest that loss of function in LAMB1 orchestrated the structural and functional plasticity of pyramidal neurons in the ACC, which in turn contributed to the development of the pain hypersensitivity and emotional aversion associated with nerve injury.

Exactly how LAMB1 conveys extracellular alterations to intracellular structural and functional plasticity and further modulates the anxiety and depression in neuropathic pain is completely unknown so far. Another interesting finding of this study is that we bridged this gap by uncovering an integrin $\beta 1$-dependent modulation of the actin cytoskeleton via the Src/RhoA/LIMK/ cofilin signaling pathway involved in the above processes. Rearrangement of the actin cytoskeleton is assumed to be associated with spine morphology and function and hence involved in various pathophysiological processes including chronic pain (32-34). Both in vitro and in vivo staining with phalloidin revealed a significant increase in polymerized F-actin in LAMB1-knockdown neurons, indicating that loss of LAMB1 leads to abnormal actin organization at dendric spines. The question then follows regarding which events link nociceptive activity-dependent downregulation of LAMB1 to actin disorganization. Integrins were first described as mediators of cellular adhesion - linking the cytoskeleton to the ECM (35). Laminins have been shown to regulate diverse cellular functions through interaction with a panel of cell-surface receptors, including different subunits of integrin and some non-integrin receptors (62-65). We wonder whether the interaction between LAMB1 and integrin may play a role in mediating the regulatory effect of LAMB1 on anxiodepression in neuropathic pain. Our immunoprecipitation assay revealed that LAMB1 interacted with integrin $\beta 1$ and that disruption of LAMB1 greatly diminished this interaction, confirming the functional interaction between LAMB1 and integrin $\beta 1$. In our examination of the downstream signaling cascades that were activated by LAMB1-integrin $\beta 1$ interaction, we found that knocking down ACC LAMB1 caused a significant reduction of $\mathrm{p}$-Src kinase, which in turn led to a reduction in expression of the RhoA-specific GAP p190RhoGap, thereby increasing the GTP-RhoA expression levels. Previous studies have reported that localized activation or recruitment of active RhoA is necessary for mushroom dendritic spine remodeling and plasticity $(40,66)$. In agreement with this scenario, we further observed that the RhoA effector ROCK2 was enhanced after LAMB1 knockdown, which further activated LIMK2 and deactivated cofilin by increasing cofilin phosphorylation. In support of our observation, RhoA has been shown to possess the ability to inhibit cofilin by phosphorylating the Ser3 residue of LIMK $(67,68)$. Since cofilin acts to sever and 
Ipsilateral
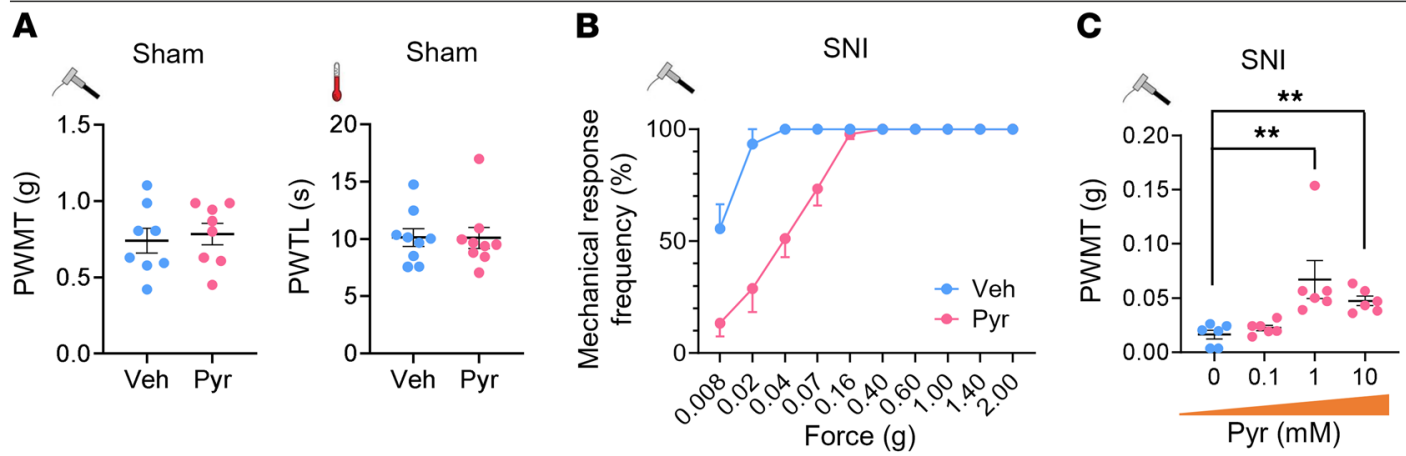

$\mathbf{E}$ EPM
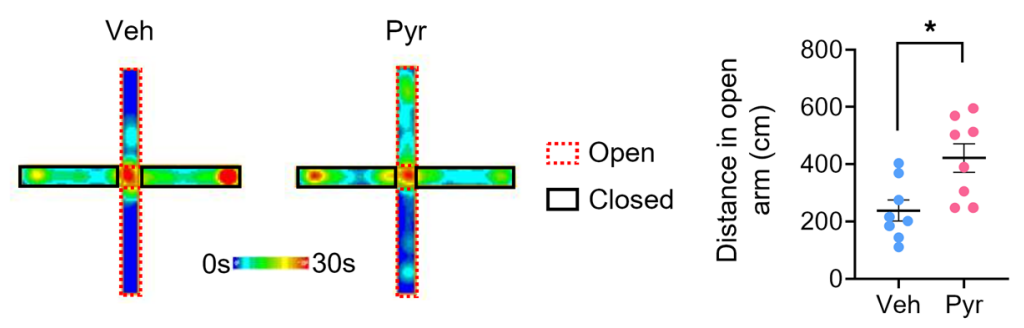

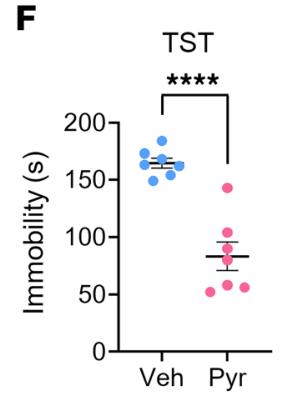

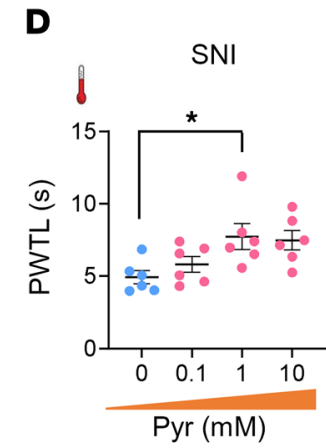

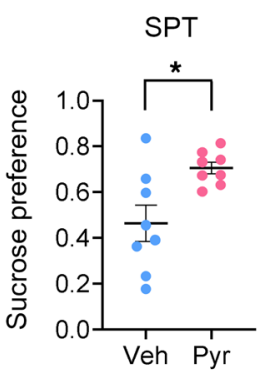

Figure 9. Intra-ACC injection of pyrintegrin, an integrin $\beta 1$ activator, relieves established pain hypersensitivity and anxiodepression after SNI. (A) Bilateral intra-ACC injection of pyrintegrin (Pyr) did not alter mechanical or thermal sensitivity in sham-treated WT mice $(n=8-9)$. Statistical significance was determined by 2-tailed, unpaired $t$ test (PWMT) and Mann-Whitney $U$ test (PWTL). (B-D) Stimulus response curves (B), mechanical threshold (C), and thermal latency (D) showing intra-ACC injection of pyrintegrin $(0.1,1,10 \mathrm{mM})$ dose-dependently relieved ipsilateral SNI-induced mechanical allodynia and thermal hyperalgesia $(n=6) .{ }^{*} P<0.05$ and ${ }^{* *} P<0.01$, by Kruskal-Wallis $H$ test with Nemenyi's multiple-comparison test (PWMT) and 1-way ANOVA with Tukey's multiple-comparison test (PWTL). (E) ACC delivery of pyrintegrin (1 mM) increased open-arm exploration by SNI-operated mice in the EPM test $(n=8) .{ }^{*} P<0.05$, by 2 -tailed, unpaired $t$ test. ( $F$ and $\left.\mathbf{G}\right)$ Intra-ACC injection of pyrintegrin $(1 \mathrm{mM})$ decreased immobility in the TST (F) and elevated sucrose preference in the SPT (C) in SNI-operated mice $(n=7-8)$. ${ }^{*} P<0.05$ and ${ }^{* * *} P<0.0001$, by 2 -tailed unpaired separate variance estimation $t$ test. Data are presented as the mean \pm SEM. See Supplemental Table 2 for detailed statistical information. Veh, vehicle.

depolymerize F-actin and increase the cellular concentration of monomeric globular actin (G-actin), inhibition of cofilin induced by LAMB1 knockdown would result in abnormal actin rearrangement and trigger spine remodeling.

Previous studies have shown that AMPAR membrane delivery and stability are dependent on the integrity of the actin cytoskeleton (48-51). In agreement with this, we detected an increased amount of the AMPAR GluR1 subunit in the plasma membrane of the ACC in LAMB1-knockdown mice as a result of actin rearrangement. We detected strong upregulation of PSD95 as well after LAMB1 knockdown. In corroboration with these results, we observed enhancement of the AMPAR-meditated synaptic response in ACC pyramidal neurons from LAMB1-knockdown mice. These lines of evidence suggest that LAMB1 downregulation-induced actin dysregulation after peripheral neuropathy leads to more AMPAR synaptic trafficking to the plasma membrane. AMPARs have emerged as important mediators of synaptic plasticity at central synapses $(52,53,56,57,69)$. AMPARs can also permit $\mathrm{Ca}^{2+}$ entry into neurons (70), and the GluR1 subunit is highly expressed in regions that have a high density of $\mathrm{Ca}^{2+}$-permeable AMPARs, including components of pain pathways $(56,71,72)$. It was thus expected that calcium photometry would show an elevation of activity of GCaMP6s-expressing pyramidal neurons in the ACC during tail suspension in LAMB1-knockdown mice. Apart from the observed increase in the GluR1 subunit in the plasma membrane, we found that LAMB1 knockdown induced membrane recruitment of the NR2A subunit as well. Although NMDAR-mediated eEPSCs and NMDA-induced currents were not significantly altered after LAMB1 knockdown, the contribution of increased NR2A expression in the plasma membrane to the plasticity of pyramidal neurons cannot be excluded. In addition to the involvement in postsynaptic plasticity, actin polymerization has also been implicated in presynaptic vesicle transport and transmitter release (73-75). In agreement with this assumption, we observed that knockdown of ACC LAMB1 induced a reduction of the PPR of AMPARs-eEPSCs and the frequency of mEPSCs in ACC pyramidal neurons. This suggests that LAMB1-mediated actin cytoskeleton rearrangement via the signaling cascades revealed here plays a crucial role in regulating presynaptic transmitter release probability. However, it will be necessary to overcome the current technical hindrances to studying the mobilization of vesicular pools in the ACC in order to better understand the mechanisms underlying LAMB1-mediated modulation of release probability at this synapse. Finally, our studies to determine whether LAMB1-mediated signaling cascades can have therapeutic effects on neuropathic pain and associated anxiodepression demonstrated that administration of the integrin $\beta 1$ activator pyrintegrin had a strong 
A
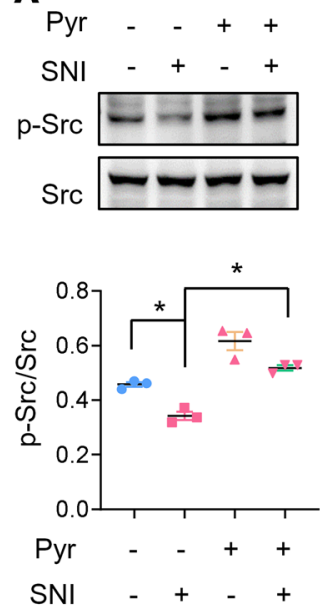

E

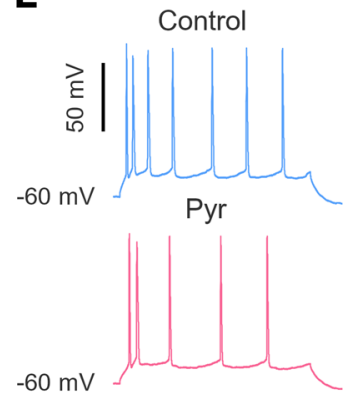

$-60 \mathrm{mV}$

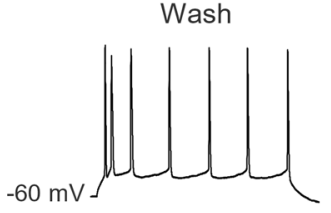

$500 \mathrm{~ms}, 100 \mathrm{pA}$
B
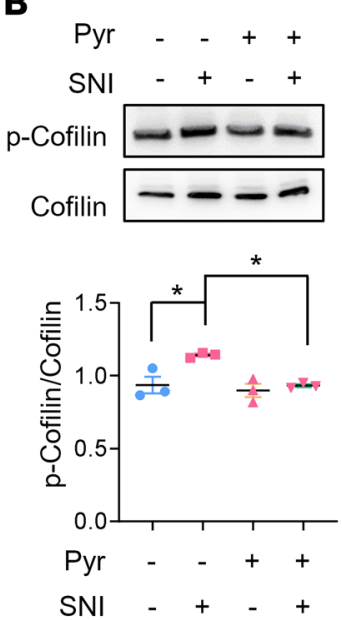

C

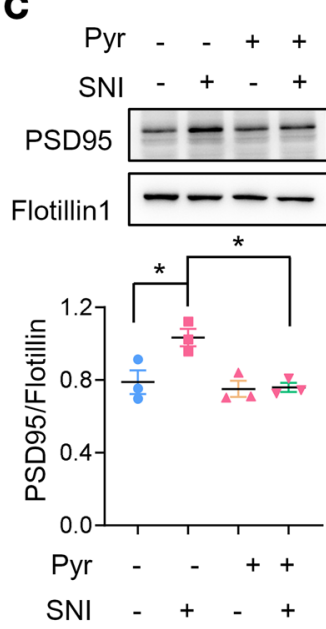

D
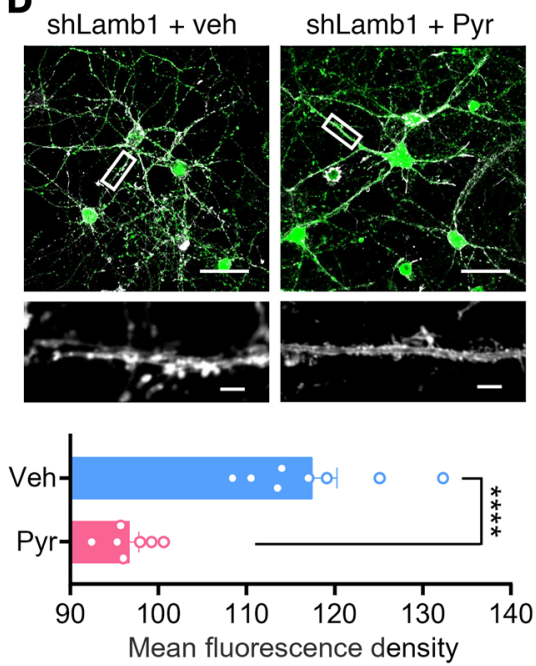

$\mathbf{F}$
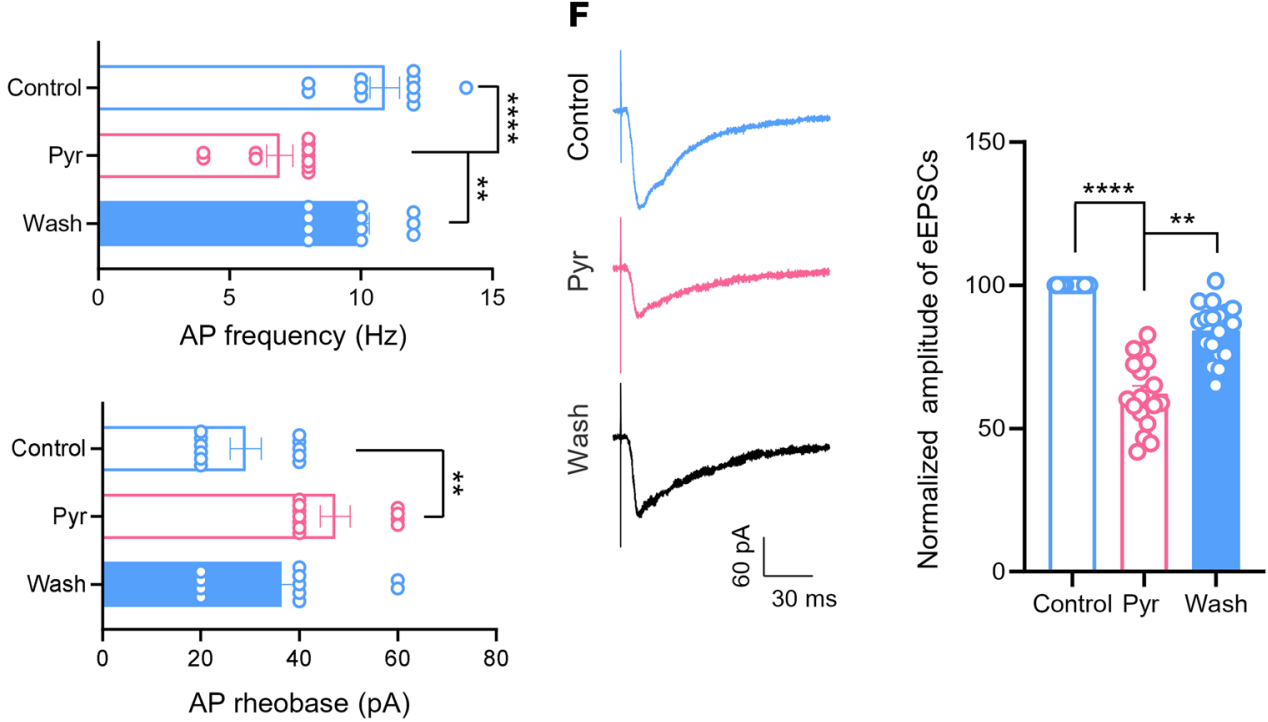

Figure 10. Pyrintegrin alleviates neuropathic pain and related aversion via the Src/cofilin signaling pathway. (A-C) Representative immunoblots and quantitative summary showing that SNI-induced decrease in p-Src (A), increase of p-cofilin (B), and PSD95 (C) was reversed by 1 mM intra-ACC pyrintegrin injection $(n=3)$. ${ }^{*} P<0.05$, by Kruskal-Wallis $H$ test with Nemenyi's multiple-comparison test ( $p$-Src/Src) and 1-way ANOVA with Tukey's multiple-comparison test ( $p$-cofilin/cofilin and PSD95/flotillin). (D) F-actin staining with phalloidin in cultured cortical neurons transfected with shLamb1 in the absence and presence of $1 \mu \mathrm{M}$ pyrintegrin ( $n=7-8$ ). Scale bars: $50 \mu \mathrm{m}$ (upper) and $5 \mu \mathrm{m}$ (lower). ${ }^{* * *} P<0.0001$, by 2-tailed, unpaired separate variance estimation $t$ test. (E and F) Bath-applied pyrintegrin $(20 \mu \mathrm{M})$ relieved neuronal hyperexcitability $(\mathbf{E})$ and AMPAR-mediated eEPSCs (F) in SNI-treated ACC pyramidal neurons $(n=11) .{ }^{* *} P<0.01$ and ${ }^{* * *} P<0.0001$, by Kruskal-Wallis $H$ test with Nemenyi's multiple-comparison test. Data are presented as the mean \pm SEM. See Supplemental Table 2 for detailed statistical information.

ability to relieve the pain hypersensitivity as well as anxiety and depression induced by SNI. In this regard, there are some limitations of this study that are worth discussing. Since the large Lamb1 gene exceeds the capacity of any regular virus vectors, such as $\mathrm{AAV}$ and lentivirus (LV), we could not investigate the impact of upregulation of LAMB1 in this study. Although we have clearly demonstrated the therapeutic effects of an integrin $\beta 1$ agonist, a CRISPR/Cas9-mediated modular RNA-guided transcriptional activation approach will need to be explored in a future study. However, in vivo implementation of the CRISPR/Cas9 gain-offunction system has proven difficult and shown limited efficacy.

In summary, this study shows how LAMB1, a key element of the ECM, conveys extracellular alterations to intracellular structural and functional plasticity and modulates neuropathic pain and anxiodepressive consequences. Furthermore, we identify pyrintegrin as a potential therapeutic candidate for the treatment of neuropathic pain and related anxiety and depression. Future studies related to aberrant remodeling of the ECM might present an important direction for chronic pain and related aversion.

\section{Methods}

\section{Animals}

Experiments were conducted in 350 adult male or female C57BL/6 mice (25-30 g), which were housed in a temperature-controlled envi- 
A

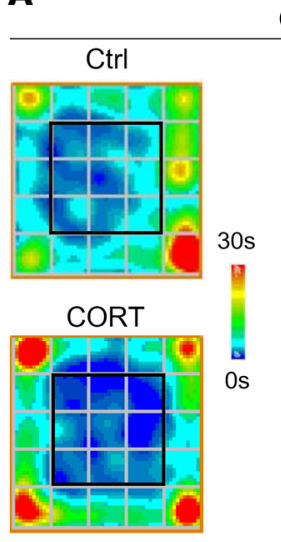

C

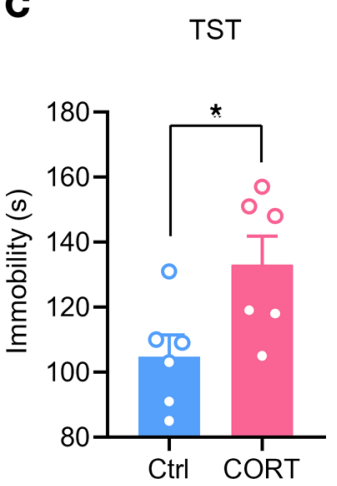

D

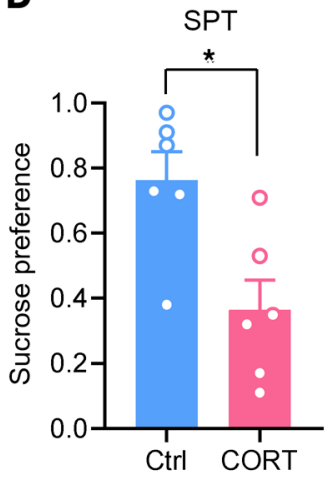

$\mathbf{F}$
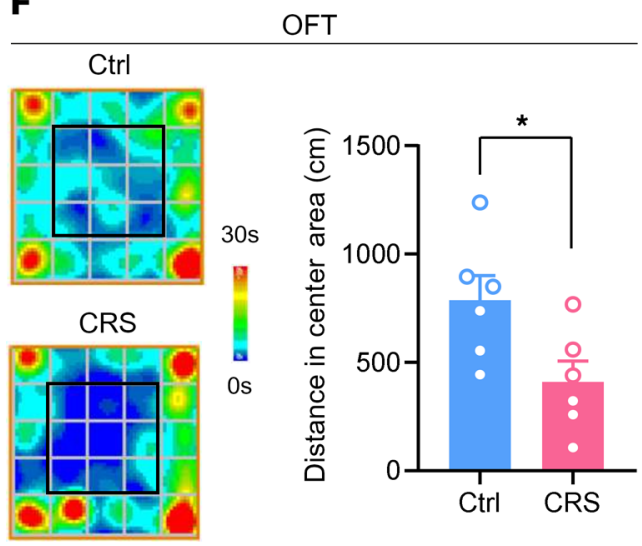

H

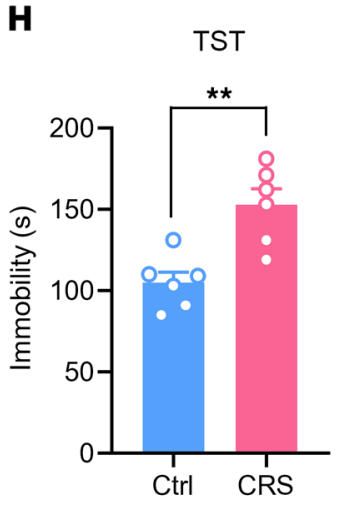

OFT

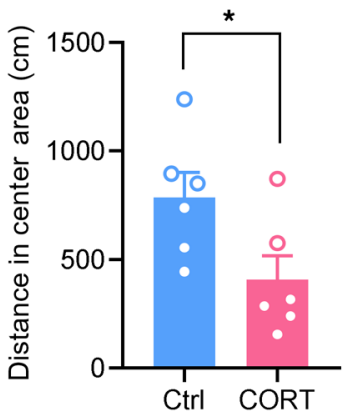

I

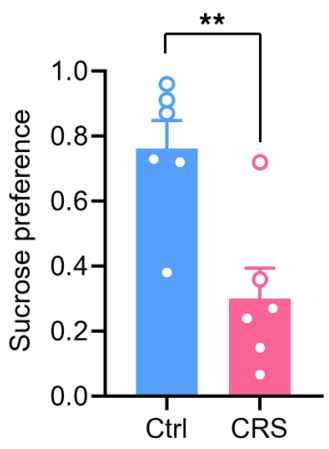

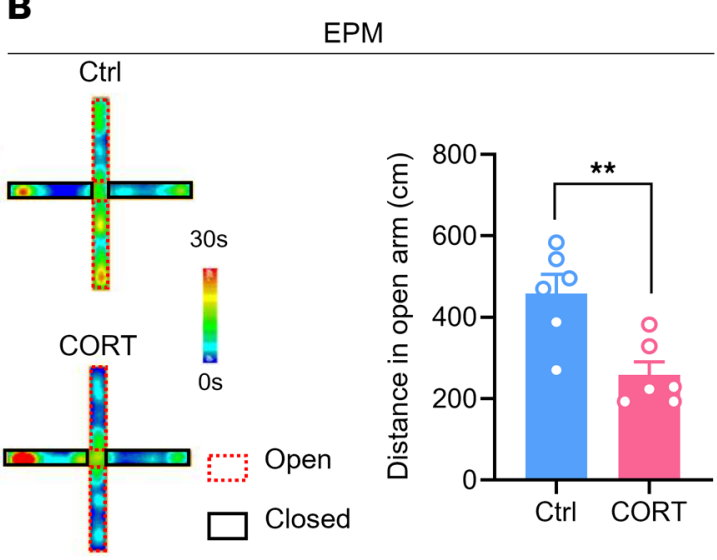

E

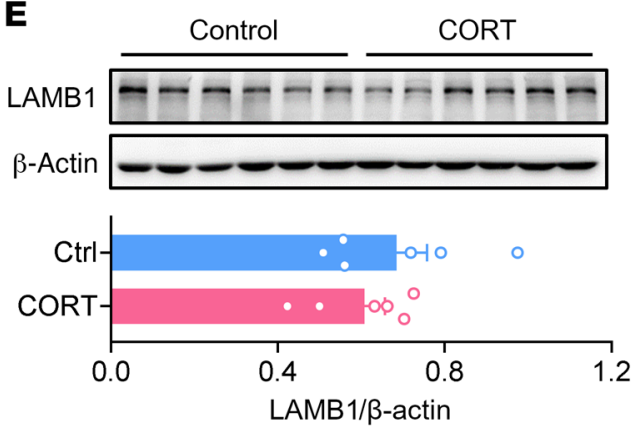

G
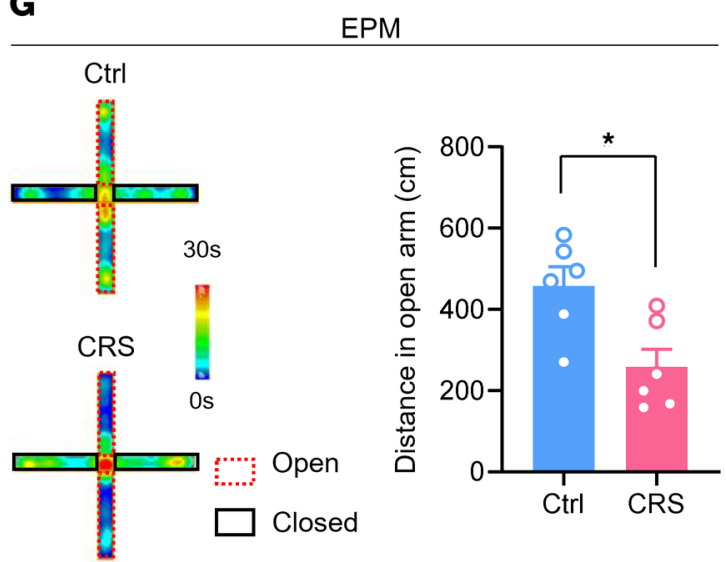

J

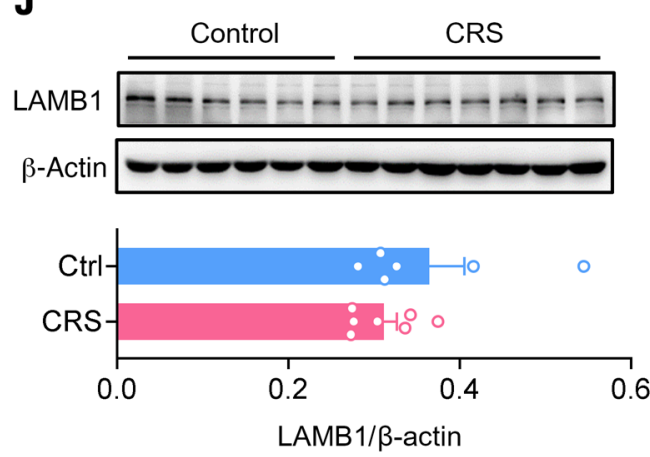


Figure 11. LAMB1 in the ACC is not involved in depression with no pain. (A and B) Representative trajectory and quantitative summary of distances traveled in the center area in the OFT (A) and in the open arm in the EPM (B) for control and CORT-treated mice $(n=6) .{ }^{*} P<0.05$ and ${ }^{*} P$ $<0.01$, by 2-tailed, unpaired $t$ test. (C and $\mathbf{D})$ Duration of immobility in the TST (C) and sucrose preference in the SPT (D) paradigm for control and CORT-treated mice $(n=6)$. ${ }^{*} P<0.05$, by 2 -tailed, unpaired $t$ test. (E) Immunoblots and quantitative summary showing that LAMB1 expression was not altered in the ACC of CORT-treated mice as compared with the control group $(n=6)$. Two-tailed, unpaired $t$ test. ( $\mathbf{F}$ and $\mathbf{C}$ ) Representative trajectory and quantitative summary of distances traveled in the center area in the OFT (F) and the open arm in the EPM (G) for control and CRS-treated mice $(n=6) .{ }^{*} P<0.05$, by 2 -tailed, unpaired $t$ test. ( $\mathbf{H}$ and $\mathbf{I}$ ) Duration of immobility in the TST $(\mathbf{H})$ and sucrose preference in the SPT (I) paradigm for control and CRS-treated mice $(n=6) .{ }^{* *} P<0.01$, by 2-tailed, unpaired $t$ test. (J) Immunoblots and quantitative summary showing that LAMB1 expression was unchanged in the ACC in CRS-treated mice as compared with the control group ( $n=6-7)$. Two-tailed unpaired separate variance estimation $t$ test. Data are presented as the mean \pm SEM. See Supplemental Table 2 for detailed statistical information.

ronment under a 12-hour light/12-hour dark cycle. Animals were randomly allocated to different experimental groups.

\section{Animal models}

SNI surgery. To mimic clinical neuropathic pain, we used a previously described SNI model (76). In brief, mice were anesthetized by i.p. injection of $1 \%$ pentasorbital sodium. The left sciatic nerve branches were isolated under aseptic surgical conditions by blunt dissection of the femoral biceps muscle. The peroneal and tibial nerves were both tightly ligated and transected distal to the ligation, leaving the sural nerve intact. The overlying muscle and skin were then sutured and sterilized after surgery. Sham-operated mice were subjected to all of the preceding procedures but without nerve ligation and transection.

Chronic inflammatory pain model. Inflammation of the paw was induced by intraplantar injection of CFA as described previously (74). See the Supplemental Methods for details.

Chronic CORT and CRS model. See the Supplemental Methods for details.

\section{Behavioral analyses}

All behavioral measurements were done in awake, unrestrained, agematched mice. Behavioral testing was carried out in habituated mice by an observer blinded to the identity of the groups. Mechanical hypersensitivity was assessed by paw withdrawal to manual application of graded von Frey hairs (0.008-2.0 g) to the plantar surface. Thermal hypersensitivity was assessed by paw withdrawal to a radiant heat source applied to the plantar surface. Anxiodepressive-like behaviors were determined with the OFT, the EPM test, the TST, and the SPT. See the Supplemental Methods for further details on the procedures.

\section{RNA-Seq}

Fifty-six days after SNI or sham surgery, the contralateral ACC was dissected under RNase-free conditions. Total RNA was isolated from the ACC using TRIzol Reagent (Thermo Fisher Scientific) in accordance with the manufacturer's protocol. Total RNA (1 $\mu$ g per sample) was used to construct sequencing libraries. Briefly, mRNA was enriched and cleaved into short fragments using a fragmentation buffer, followed by reverse transcription into cDNA using random primers. The
cDNA fragments were purified and end repaired, and a poly(A) tail was added, followed by ligation with Illumina sequencing adapters. The ligation products were then size selected by agarose gel electrophoresis, amplified by PCR, and sequenced using the Illumina Novaseq 6000 (Gene Denovo Biotechnology). DEGs were assessed by analysis of differential RNA expression between 2 groups. Transcripts with the parameter of a FDR below 0.05 and an absolute fold change of 2 or greater were considered differentially expressed. Pathway enrichment analysis was performed using the KEGG. The calculated $P$ value was FDR corrected, with an FDR of 0.05 or less as a threshold. Pathways meeting this condition were defined as significantly enriched pathways in DEGs. The RNA-Seq data used in this study have been deposited in the NCBI's Sequence Read Archive (SRA) (SRA study accession code, SRP323752; https://www.ncbi.nlm.nih.gov/Traces/study/?acc=PRJNA736747\&o=acc_s\%3Aa4).

qRT-PCR. See the Supplemental Methods for details, including the primers and probes used, which are listed in Supplemental Table 1.

\section{Western blot analysis and immunofluorescence labeling}

See the Supplemental Methods for details, including the list of antibodies used in Supplemental Table 1.

\section{Coimmunoprecipitation assay}

Three weeks after intracingulate injection of AAV2/9-U6-Lamb1 shRNA-CMV-EGFP (AAV-shLamb1) or AAV2/9-U6-scrambled shRNA-CMV-EGFP (AAV-scrambled shRNA), ACC tissues were lysed on ice for 30 minutes in immunoprecipitation RIPA buffer supplemented with the protease inhibitor PMSF, centrifuged at 13,680 $g$ for 10 minutes, and immunoprecipitated with Protein A/G PLUS Agarose Immunoprecipitation Reagent (YT-883, Beijing BAIAOLAIBO) according to the manufacturer's instructions. Briefly, lysates were isolated $40 \mu \mathrm{L}$ for input as a positive control, and others were precleared by incubating on ice for 30 minutes with $1 \mu \mathrm{g}$ control IgG and $20 \mu \mathrm{L}$ Protein A/G PLUS Agarose. Beads were pelleted by centrifugation at $590 \mathrm{~g}$ for 5 minutes at $4^{\circ} \mathrm{C}$. The resulting supernatant (approximately $500-1000 \mu \mathrm{g}$ total protein) was incubated with $2 \mu \mathrm{g}$ primary antibody (Supplemental Table 1;2 $2 \mu \mathrm{g}$ IgG was used as a negative control) overnight at $4^{\circ} \mathrm{C}$, and then with $40 \mu \mathrm{L}$ Protein A/G PLUS Agarose for 4 hours at $4^{\circ} \mathrm{C}$. Immunoprecipitates were collected by centrifugation at $590 \mathrm{~g}$ for 5 minutes at $4^{\circ} \mathrm{C}$, washed 4 times with immunoprecipitation RIPA buffer, resuspended in $40 \mu \mathrm{L} 1 \times$ loading buffer, and then boiled for 5 minutes. Samples $(10 \mu \mathrm{L})$ were then resolved by SDS-PAGE and immunoblotted with the appropriate antibodies.

\section{Rho activation assay}

Three weeks following intracingulate injection of AAV-shLamb1 or AAV-scrambled shRNA, ACC tissue was lysed on ice for 30 minutes in cell lysis buffer supplemented with protease inhibitor cocktail and centrifuged at 13,680 g for 10 minutes. A 20-50 $\mu \mathrm{g}$ sample was kept for Western blot quantitation of total RhoA per sample. Total protein (800 $\mu \mathrm{g}$ ) was measured by pulldown assay (BK036, Cytoskeleton) according to the manufacturer's instructions. Briefly, $50 \mu \mathrm{g}$ rhotekin-RBD beads was added to the lysates and incubated at $4^{\circ} \mathrm{C}$ on a rotator for 1 hour. Rhotekin-RBD beads were then pelleted by centrifugation at $5000 \mathrm{~g}$ at $4^{\circ} \mathrm{C}$ for 1 minute, and the supernatants were carefully removed. Laemmli $2 \times$ sample buffer $(20 \mu \mathrm{L})$ was added to each tube, and the bead samples were boiled for 2 minutes. The samples were then analyzed by SDS-PAGE and Western blotting. 


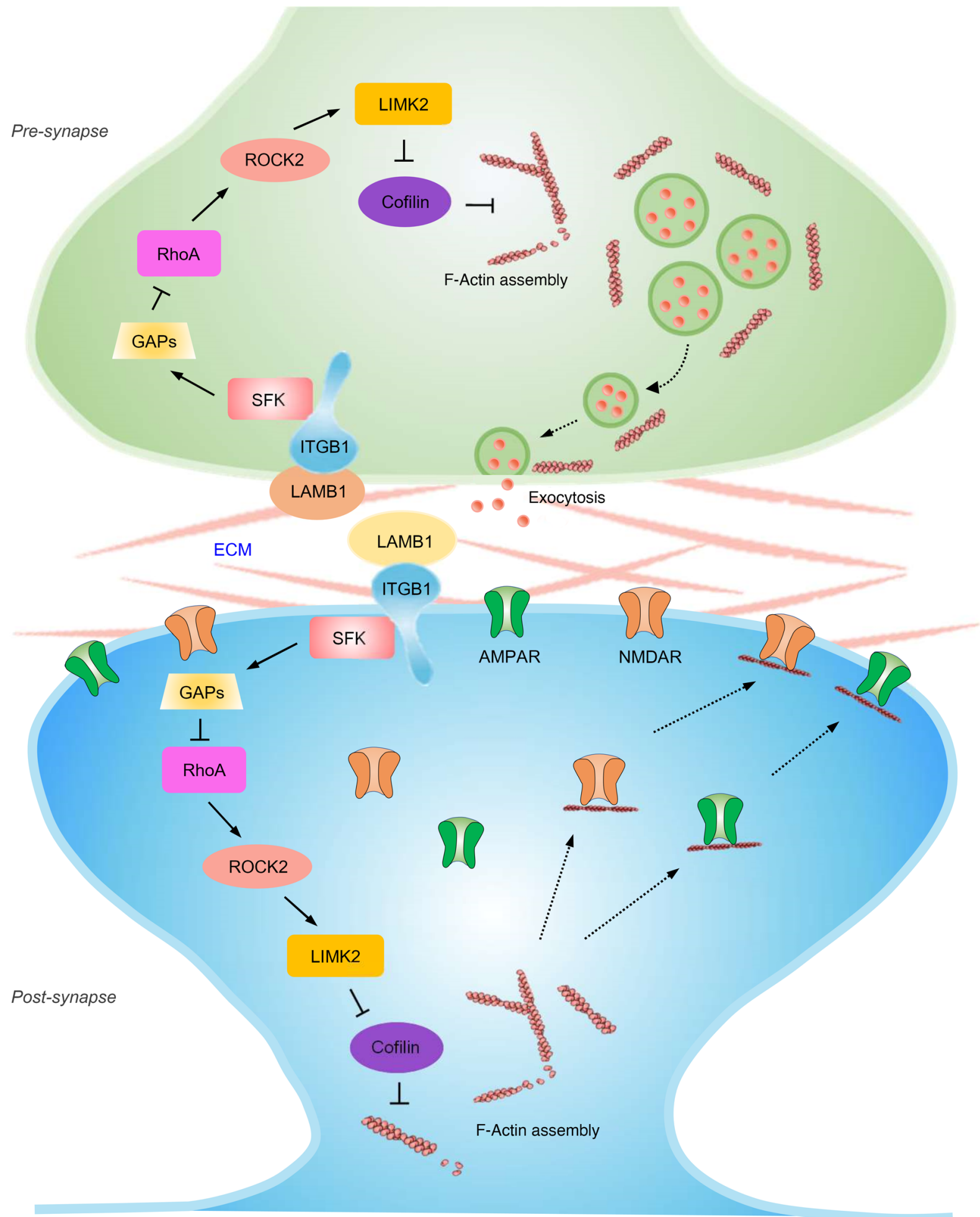

Figure 12. Pre- and post-synapse model. Schematic model proposing how LAMB1, a key element of the ECM, conveys extracellular alterations to intracellular structural and functional plasticity and modulates neuropathic pain and pain-related anxiodepression. See the text for details.

\section{Golgi staining}

Golgi-Cox staining was performed as described in detail previously $(77,78)$. Stack images were captured using an Olympus FV1200 confocal microscope, and Imaris software (version 7.7.1, Bitplane) was used to reconstruct neuronal dendritic arbors and dendritic spines.
Dendritic length, branching complexity, and branch-point locations in a series of concentric circles (at $10 \mu \mathrm{m}$ radius increments from the soma) throughout the whole dendritic arbor were quantified for Sholl analysis. See the Supplemental Methods for details, including the reagents used, which are listed in Supplemental Table 1. 


\section{Stereotaxic surgery}

See the Supplemental Methods for details, including the virus used (Supplemental Table 1).

\section{Brain slice patch-clamp recording}

A whole-cell patch-clamp recording was performed as described previously (79). Briefly, 3 weeks after viral injection of AAV-shLamb1 or AAV-scrambled shRNA into the unilateral ACC, mice were anesthetized with urethane $(1.2 \mathrm{~g} / \mathrm{kg}$, i.p.) and transcardially perfused with ice-cold oxygenated $\left(95 \% \mathrm{O}_{2}, 5 \% \mathrm{CO}_{2}\right)$ incubation solution $(95 \mathrm{mM}$ $\mathrm{NaCl}, 1.8 \mathrm{mM} \mathrm{KCl}, 1.2 \mathrm{mM} \mathrm{KH}_{2} \mathrm{PO}_{4}, 0.5 \mathrm{mM} \mathrm{CaCl}_{2}, 7 \mathrm{mM} \mathrm{MgSO}_{4}, 26$ $\mathrm{mM} \mathrm{NaHCO}_{3}, 15 \mathrm{mM}$ glucose, $50 \mathrm{mM}$ sucrose, $\mathrm{pH}$ 7.4). Coronal slices (300 $\mu \mathrm{m}$ thick) containing the injected ACC were prepared using the vibratome and stored in an incubation solution at $32 \pm 1^{\circ} \mathrm{C}$ for at least 1 hour. Standard whole-cell patch clamp recordings were performed in fluorescence-labeled pyramidal neurons in layers II and III of the ACC in a recording solution, which was identical to the incubation solution except for the following: $127 \mathrm{mM} \mathrm{NaCl}, 2.4 \mathrm{mM} \mathrm{CaCl}_{2}, 1.3$ $\mathrm{mM} \mathrm{MgSO}_{4}$, and $0 \mathrm{mM}$ sucrose. The pipette solution consisted of $135 \mathrm{mM}$ K-gluconate, $5 \mathrm{mM} \mathrm{KCl}, 0.5 \mathrm{mM} \mathrm{CaCl}, 2 \mathrm{mM} \mathrm{MgCl}_{2}, 5 \mathrm{mM}$ EGTA, 5 mM HEPES, and $5 \mathrm{mM} \mathrm{Mg-ATP,} \mathrm{pH} \mathrm{7.4,} \mathrm{with} \mathrm{KOH,} \mathrm{at} \mathrm{a} \mathrm{mea-}$ sured osmolarity of $300 \mathrm{mOsm}$. The electrophysiological properties of the recorded neurons were acquired with an Axon 700B amplifier and pCLAMP 10 software (both from Molecular Devices). Except for the AP recording, $5 \mathrm{mM} \mathrm{QX-314}$ was added to the pipette solution to prevent AP discharge. Data were excluded when the resting membrane potential of neurons was above $-55 \mathrm{mV}$ and the AP did not have overshoot. Signals were low-pass filtered at $5 \mathrm{kHz}$, sampled at $10 \mathrm{kHz}$, and analyzed offline.

Input-output of AMPAR-mediated eEPSCs. The cell membrane potential was held at $-70 \mathrm{mV}$. AMPARs-eEPSCs were recorded from layer II/III neurons, and the stimulations were delivered by a field-stimulating electrode placed in layers V and VI of the ACC. AMPARs-eEPSCs were induced by repetitive stimulations at $0.05 \mathrm{~Hz}$, with increasing intensity in the presence of AP5 $(50 \mu \mathrm{M})$ and picrotoxin $(100 \mu \mathrm{M})$.

AMPAR/NMDAR ratio. The AMPAR- and NMDAR-mediated current ratio was recorded in the presence of $100 \mu \mathrm{M}$ picrotoxin at holding potentials of $-70 \mathrm{mV}$ and $+40 \mathrm{mV}$ with an internal solution containing the following: $107 \mathrm{mM} \mathrm{CsMeSO}{ }_{3}, 10 \mathrm{mM} \mathrm{CsCl}_{2}, 3.7 \mathrm{mM}$ $\mathrm{NaCl}, 5$ mM TEA-Cl, 20 mM HEPES, 0.2 mM EGTA, 5 mM QX-314, 4 mM ATP magnesium salt, $0.3 \mathrm{mM}$ GTP sodium salt, and $2 \mathrm{mM} \mathrm{MgCl}_{2}$. The AMPAR/NMDAR ratio was calculated as the ratio of the average EPSC peak amplitude at $-70 \mathrm{mV}$ to that recorded at $+40 \mathrm{mV}$ (average of 40-50 ms after afferent stimulation).

PPR analysis. In a subset of experiments, paired-pulse stimuli with an interstimulus interval of $50 \mathrm{~ms}(0.1 \mathrm{~ms}$ pulse duration, $1 \mathrm{~mA}$ intensity, every 30 seconds) were applied to layers V and VI of the ACC. The PPR (facilitation or depression) of AMPARs-eEPSCs was calculated as the amplitude of the second eEPSC divided by that of the first eEPSC in a pair.

Intrinsic membrane properties. For analysis of membrane properties, the membrane potential was held at $-70 \mathrm{mV}$ under current-clamp mode. Depolarizing current steps (500 ms in duration, 20 pA increments) were used to detect the AP. The AP threshold was determined by differentiating the AP waveform and setting a rising rate of $10 \mathrm{mV} /$ $\mathrm{ms}$ as the AP inflection point. The AP amplitude was measured from the equipotential point of the threshold to the spike peak. The AP duration was measured at the half-width of the spike.

AMPA- or NMDA-induced current recordings. AMPA-induced currents were recorded while $50 \mu \mathrm{M}$ AMPA was bath applied for 30 seconds at a holding potential of $-70 \mathrm{mV}$, and NMDA-induced currents were recorded while $50 \mu \mathrm{M}$ NMDA was bath applied for 30 seconds at $+40 \mathrm{mV}$.

Analysis of mEPSCs. mEPSCs were recorded at a holding potential of $-70 \mathrm{mV}$ in the presence of AP5 $(50 \mu \mathrm{M})$, picrotoxin $(100 \mu \mathrm{M})$, and tetrodotoxin (TTX) $(0.5 \mu \mathrm{M})$. mEPSCs were analyzed with Mini Analysis software (Synaptosoft).

Pyrintegrin effect. In a subset of experiments, $20 \mu \mathrm{M}$ pyrintegrin was applied by bath superfusion for 30 seconds to assess its role in the hyperexcitability and synaptic transmission of ACC pyramidal neurons derived from SNI-treated mice. Membrane properties and AMPARs-eEPSCs were examined before, during, and after pyrintegrin application.

\section{Fiber photometry}

Fiber photometry was used to record calcium-dependent activity dynamics during tail suspension with the ThinkerTech fiber photometry system as described previously (80). An optical fiber was implanted into the ACC of mice 3 weeks after combined viral injection of AAVCaMKII-GCaMP6s with AAV-shLamb1 or AAV-scrambled shRNA, as described above. To excite GCaMP6s, a 473 nm LED (Cree XP-E LED) was reflected off a dichroic mirror (MD498, Thorlabs) that was focused using a $0.4 \mathrm{NA} \times 20$ objective lens (Olympus) and coupled to an optical commutator (Doris Lenses). An optical fiber (230 $\mu \mathrm{m}$ OD, $0.37 \mathrm{NA}$ ) guided the light between the commutator and the implanted optical fiber. The laser power at the tip of the optical fiber was adjusted to $0.01-0.02 \mathrm{~mW}$ to decrease laser bleaching. Fluorescence was bandpass filtered (MF525-39, Thorlabs), and an amplifier was used to convert the CMOS (DCC3240M, Thorlabs) current output to signals, which were further filtered through a low-pass filter $(40 \mathrm{~Hz}$ cutoff, ThinkerTech). The analog voltage signals were digitalized at $50 \mathrm{~Hz}$ and recorded by the multichannel fiber photometry recording system (ThinkerTech). Fluorescence signals were recorded continuously during tail suspension and normalized $(\Delta \mathrm{F} / \mathrm{F})$ by calculating the median signal across the recording period, subtracting this from each data point, and dividing by the median signal.

\section{Pharmacological rescue experiments}

The ACC from sham-treated or 8-week post-SNI mice was implanted with a bilateral 26 gauge stainless steel guide cannula. After recovery from surgery for approximately 7 days, $600 \mathrm{~nL}$ pyrintegrin $(0.1,1$, or $10 \mathrm{mM}$ ) or $0.9 \%$ saline as vehicle was infused into each side over a 2-minute period. Behavioral tests were performed approximately 1 hour after microinjection. See the Supplemental Methods for more detailed procedures, including the list of reagents used in Supplemental Table 1.

\section{Cortical cell culture}

See the Supplemental Methods for details, including the list of reagents used in Supplemental Table 1.

\section{Statistics}

Data were analyzed using GraphPad Prism, version 8.0 (GraphPad Software) and SPSS 21.0 software. The normality test was performed 
with the Shapiro-Wilk test. The homogeneity of variance test was performed using Levene's test. Data that met these 2 conditions were analyzed using a 2-tailed, unpaired or paired $t$ test, a 1-way ANOVA, or a repeated-measures ANOVA followed by Tukey's multiple-comparison test. Data sets that were not normally distributed were analyzed with a nonparametric test (Supplemental Table 2). Data are reported as the mean \pm SEM. A $P$ value of less than 0.05 was considered statistically significant.

\section{Study approval}

All animal procedures were reviewed and approved by the IACUC of the Fourth Military Medical University (FMMU) in Xi'an, China. All testing was conducted in a double-blinded manner.

\section{Author contributions}

ZZL, WNL, and TZW conducted Western blot analyses. WJH and FW performed brain slice patch-clamp recordings. ZZL, YC, JYS, and WNL performed animal preparation. ZZL, YC, and JYS performed and analyzed the Golgi staining. JYS and YDX performed immunofluorescence staining. ZZL, YC, JYS, and WNL conducted behavioral and pharmacological testing. ZZL, ZCS, and FDW conducted stereotaxic surgery and fiber photometry. ZZL and GHC designed and performed the RNA-Seq analysis. ZZL, HHM, WJH, SBM, ZCS, and RGX analyzed data. CL, SXW, and ZZL designed studies. CL and ZZL wrote the draft of the manuscript. CL and SXW supervised the experiments and revised the manuscript. All the authors read and approved the final manuscript.

\section{Acknowledgments}

This work was supported by grants from the Natural Science Foundation of China (NSFC) (32071002 and 31671088, to CL; 81730035 , to SXW; and 81870867, to RGX).

Address correspondence to: Sheng-Xi Wu or Ceng Luo, Department of Neurobiology, School of Basic Medicine, Fourth Military Medical University, Changle West Road no. 169, Xian, Shaanxi Province 710032, China. Phone: 86.29.84712312; Email: shengxi@fmmu.edu.cn (SXW); Email: luoceng@fmmu. edu.cn (CL).
1. Campbell JN, Meyer RA. Mechanisms of neuropathic pain. Neuron. 2006;52(1):77-92.

2. Bair MJ, et al. Depression and pain comorbidity: a literature review. Arch Intern Med. 2003;163(20):2433-2445.

3. McWilliams LA, et al. Depression and anxiety associated with three pain conditions: results from a nationally representative sample. Pain. 2004;111(1-2):77-83.

4. Simon GE, et al. An international study of the relation between somatic symptoms and depression. N Engl J Med. 1999;341(18):1329-1335.

5. Arnow BA, et al. Comorbid depression, chronic pain, and disability in primary care. Psychosom Med. 2006;68(2):262-268.

6. Goyal M, et al. Meditation programs for psychological stress and well-being: a systematic review and meta-analysis. JAMA Intern Med. 2014;174(3):357-368.

7. Campbell LC, et al. Persistent pain and depression: a biopsychosocial perspective. Biol Psychiatry. 2003;54(3):399-409.

8. Damsa C, et al. Current status of brain imaging in anxiety disorders. Curr Opin Psychiatry. 2009;22(1):96-110.

9. Latremoliere A, Woolf CJ. Central sensitization: a generator of pain hypersensitivity by central neural plasticity. J Pain. 2009;10(9):895-926.

10. Vogt BA. Pain and emotion interactions in subregions of the cingulate gyrus. Nat Rev Neurosci. 2005;6(7):533-544.

11. Barthas F, et al. The anterior cingulate cortex is a critical hub for pain-induced depression. Biol Psychiatry. 2015;77(3):236-245.

12. Apkarian AV, et al. Human brain mechanisms of pain perception and regulation in health and disease. Eur J Pain. 2005;9(4):463-484.

13. Bliss TV, et al. Synaptic plasticity in the anterior cingulate cortex in acute and chronic pain. Nat Rev Neurosci. 2016;17(8):485-496.

14. Koga K, et al. Coexistence of two forms of LTP in ACC provides a synaptic mechanism for the interactions between anxiety and chronic pain. Neuron. 2015;85(2):377-389.

15. Zhuo M. Neural mechanisms underlying anxietychronic pain interactions. Trends Neurosci. 2016;39(3):136-145.

16. Zhuo M. Long-term potentiation in the anterior cingulate cortex and chronic pain. Philos Trans $R$ Soc Lond B Biol Sci. 2014;369(1633):20130146.

17. Sellmeijer J, et al. Hyperactivity of anterior cingulate cortex areas $24 \mathrm{a} / 24 \mathrm{~b}$ drives chronic pain-induced anxiodepressive-like consequences. JNeurosci. 2018;38(12):3102-3115.

18. Dityatev A, et al. The dual role of the extracellular matrix in synaptic plasticity and homeostasis. Nat Rev Neurosci. 2010;11(11):735-746.

19. Tajerian M, et al. The hippocampal extracellular matrix regulates pain and memory after injury. Mol Psychiatry. 2018;23(12):2302-2313.

20. Colognato H, Yurchenco PD. Form and function: the laminin family of heterotrimers. Dev Dyn. 2000;218(2):213-234.

21. Aumailley M, et al. A simplified laminin nomenclature. Matrix Biol. 2005;24(5):326-332.

22. Indyk JA, et al. Laminin chain expression suggests that laminin-10 is a major isoform in the mouse hippocampus and is degraded by the tissue plasminogen activator/plasmin protease cascade during excitotoxic injury. Neuroscience. 2003;116(2):359-371.

23. Sharif KA, et al. Differential regulation of laminin b1 transgene expression in the neonatal and adult mouse brain. Neuroscience. 2004;126(4):967-978.

24. Laifenfeld D, et al. Antidepressants and prolonged stress in rats modulate CAM-L1, laminin, and PCREB, implicated in neuronal plasticity. Neurobiol Dis. 2005;20(2):432-441.

25. Laifenfeld D, et al. Alterations in cell adhesion molecule L1 and functionally related genes in major depression: a postmortem study. Biol Psychiatry. 2005;57(7):716-725.

26. Palu E, Liesi P. Differential distribution of laminins in Alzheimer disease and nor- mal human brain tissue. J Neurosci Res. 2002;69(2):243-256.

27. Yang YC, et al. Laminin- $\beta 1$ impairs spatial learning through inhibition of ERK/MAPK and SGK1 signaling. Neuropsychopharmacology. 2011;36(12):2571-2586.

28. Pantazopoulos H, Berretta S. In sickness and in health: perineuronal nets and synaptic plasticity in psychiatric disorders. Neural Plast. 2016;2016:9847696.

29. Lubbers BR, et al. Neural ECM in addiction, schizophrenia, and mood disorder. Prog Brain Res. 2014;214:263-284.

30. Tsvetkov E, et al. Fear conditioning occludes LTP-induced presynaptic enhancement of synaptic transmission in the cortical pathway to the lateral amygdala. Neuron. 2002;34(2):289-300.

31. Schulz PE, et al. Using paired-pulse facilitation to probe the mechanisms for long-term potentiation (LTP). J Physiol Paris. 1995;89(1):3-9.

32. Cingolani LA, Goda Y. Actin in action: the interplay between the actin cytoskeleton and synaptic efficacy. Nat Rev Neurosci. 2008;9(5):344-356.

33. Fukazawa Y, et al. Hippocampal LTP is accompanied by enhanced F-actin content within the dendritic spine that is essential for late LTP maintenance in vivo. Neuron. 2003;38(3):447-460.

34. Li Y, et al. LIMK-dependent actin polymerization in primary sensory neurons promotes the development of inflammatory heat hyperalgesia in rats. Sci Signal. 2014;7(331):ra61.

35. Miranti CK, Brugge JS. Sensing the environment: a historical perspective on integrin signal transduction. Nat Cell Biol. 2002;4(4):E83-E90.

36. Relucio J, et al. Laminin alters fyn regulatory mechanisms and promotes oligodendrocyte development. J Neurosci. 2009; 29(38):11794-11806.

37. Cabodi $S$, et al. Integrin signaling adaptors: not only figurants in the cancer story. Nat Rev Cancer. 2010;10(12):858-870.

38. Arthur WT, et al. Integrin engagement suppresses 
RhoA activity via a c-Src-dependent mechanism. Curr Biol. 2000;10(12):719-722.

39. Lin WH, Webb DJ. Actin and actin-binding proteins: masters of dendritic spine formation, morphology, and function. Open Neurosci J. 2009;3:54-66.

40. Murakoshi H, et al. Local, persistent activation of Rho GTPases during plasticity of single dendritic spines. Nature. 2011;472(7341):100-104.

41. Pontrello CG, Ethell IM. Accelerators, brakes, and gears of actin dynamics in dendritic spines. Open Neurosci J. 2009;3:67-86.

42. Shi Y, et al. Focal adhesion kinase acts downstream of EphB receptors to maintain mature dendritic spines by regulating cofilin activity. J Neurosci. 2009;29(25):8129-8142.

43. Pontrello CG, et al. Cofilin under control of $\beta$-arrestin-2 in NMDA-dependent dendritic spine plasticity, long-term depression (LTD), and learning. Proc Natl Acad Sci U S A. 2012;109(7):E442-E451.

44. Brouns MR, et al. p190 RhoGAP is the principal Src substrate in brain and regulates axon outgrowth, guidance and fasciculation. Nat Cell Biol. 2001;3(4):361-367.

45. Tamura H, et al. Protein tyrosine phosphatase receptor type $\mathrm{Z}$ is involved in hippocampusdependent memory formation through dephosphorylation at Y1105 on p190 RhoGAP. Neurosci Lett. 2006;399(1-2):33-38.

46. Sfakianos MK, et al. Inhibition of Rho via Arg and p190RhoGAP in the postnatal mouse hippocampus regulates dendritic spine maturation, synapse and dendrite stability, and behavior. J Neurosci. 2007;27(41):10982-10992.

47. dos Remedios CG, et al. Actin binding proteins: regulation of cytoskeletal microfilaments. Physiol Rev. 2003;83(2):433-473.

48. Hanley JG. Actin-dependent mechanisms in AMPA receptor trafficking. Front Cell Neurosci. 2014;8:381.

49. Gu J, et al. ADF/cofilin-mediated actin dynamics regulate AMPA receptor trafficking during synaptic plasticity. Nat Neurosci. 2010;13(10):1208-1215.

50. Rocca DL, et al. Inhibition of Arp2/3-mediated actin polymerization by PICK1 regulates neuronal morphology and AMPA receptor endocytosis. Nat Cell Biol. 2008;10(3):259-271.

51. Yuen EY, et al. Regulation of AMPA receptor channels and synaptic plasticity by cofilin phosphatase Slingshot in cortical neurons. JPhysiol. 2010;588(pt 13):2361-2371.

52. Toyoda H, et al. Time-dependent postsynaptic AMPA GluR1 receptor recruitment in the cingulate synaptic potentiation. Dev Neurobiol. 2007;67(4):498-509.

53. Toyoda H, et al. Roles of the AMPA receptor subunit GluA1 but not GluA2 in synaptic potentiation and activation of ERK in the anterior cingulate cortex. Mol Pain. 2009;5:46.

54. Hayashi Y, et al. Driving AMPA receptors into synapses by LTP and CaMKII: requirement for GluR1 and PDZ domain interaction. Science. 2000;287(5461):2262-2267.

55. Yang Y, et al. Delivery of AMPA receptors to perisynaptic sites precedes the full expression of long-term potentiation. Proc Natl Acad Sci U S A. 2008;105(32):11388-11393.

56. Hartmann B, et al. The AMPA receptor subunits GluR-A and GluR-B reciprocally modulate spinal synaptic plasticity and inflammatory pain. Neuron. 2004;44(4):637-650.

57. Malinow R. AMPA receptor trafficking and longterm potentiation. Philos Trans R Soc Lond B Biol Sci. 2003;358(1432):707-714.

58. Shi S, et al. Subunit-specific rules governing AMPA receptor trafficking to synapses in hippocampal pyramidal neurons. Cell. 2001;105(3):331-343.

59. Wu Y, et al. Expression of laminin beta1 in hippocampi of patients with intractable epilepsy. Neurosci Lett. 2008;443(3):160-164.

60. Wu Y, et al. Expression of laminin $\beta 1$ and integrin $\alpha 2$ in the anterior temporal neocortex tissue of patients with intractable epilepsy. Int J Neurosci. 2011;121(6):323-328.

61. Kuner R, Flor H. Structural plasticity and reorganisation in chronic pain. Nat Rev Neurosci. 2017;18(2):113.

62. Belkin AM, Stepp MA. Integrins as receptors for laminins. Microsc Res Tech. 2000;51(3):280-301.

63. Plantman S, et al. Integrin-laminin interactions controling neurite outgrowth from adult DRG neurons in vitro. Mol Cell Neurosci. 2008;39(1):50-62.

64. Yamada M, Sekiguchi K. Molecular basis of laminin-integrin interactions. Curr Top Membr. 2015;76:197-229.

65. Cloutier G, et al. Non-integrin laminin receptors in epithelia. Tissue Cell. 2019;56:71-78.

66. Schubert V, et al. Localized recruitment and activation of RhoA underlies dendritic spine morphology in a glutamate receptor-dependent manner. J Cell Biol. 2006;172(3):453-467.

67. Ohashi K, et al. Rho-associated kinase ROCK activates LIM-kinase 1 by phosphorylation at threonine 508 within the activation loop. J Biol Chem. 2000;275(5):3577-3582.

68. Yang N, et al. Cofilin phosphorylation by LIMkinase 1 and its role in Rac-mediated actin reorganization. Nature. 1998;393(6687):809-812.

69. Huganir RL, Nicoll RA. AMPARs and synaptic plasticity: the last 25 years. Neuron. 2013;80(3):704-717.

70. Burnashev N, et al. Divalent ion permeability of AMPA receptor channels is dominated by the edited form of a single subunit. Neuron. 1992;8(1):189-198.

71. Engelman HS, et al. The distribution of neurons expressing calcium-permeable AMPA receptors in the superficial laminae of the spinal cord dorsal horn. J Neurosci. 1999;19(6):2081-2089.

72. Descalzi G, et al. Rapid synaptic potentiation within the anterior cingulate cortex mediates trace fear learning. Mol Brain. 2012;5:6.

73. Cole JC, et al. Disruption of actin impedes transmitter release in snake motor terminals. J Physiol. 2000;525 Pt 3:579-586.

74. Sankaranarayanan S, et al. Actin has a molecular scaffolding, not propulsive, role in presynaptic function. Nat Neurosci. 2003;6(2):127-135.

75. Rust MB, Maritzen T. Relevance of presynaptic actin dynamics for synapse function and mouse behavior. Exp Cell Res. 2015;335(2):165-171.

76. Decosterd I, Woolf CJ. Spared nerve injury: an animal model of persistent peripheral neuropathic pain. Pain. 2000;87(2):149-158.

77. Lu J, et al. A role for Kalirin-7 in nociceptive sensitization via activity-dependent modulation of spinal synapses. Nat Commun. 2015;6:6820.

78. Simonetti $\mathrm{M}$, et al. Nuclear calcium signaling in spinal neurons drives a genomic program required for persistent inflammatory pain. Neuron. 2013;77(1):43-57.

79. Wang F, et al. Nociceptor-localized cGMPdependent protein kinase I is a critical generator for central sensitization and neuropathic pain. Pain. 2021;162(1):135-151.

80. Guo B, et al. Anterior cingulate cortex dysfunction underlies social deficits in Shank3 mutant mice. Nat Neurosci. 2019;22(8):1223-1234. 